\title{
On Equatorial Dynamics, Mixed Layer Physics and Sea Surface Temperature
}

\author{
PAUL S. SCHOPF \\ Goddard Laboratory for Atmospheric Sciences, NASA/Goddard Space Flight Center, Greenbelt MD 20771 \\ Mark A. CANE \\ Department of Meteorology and Physical Oceanography, MIT, Cambridge, MA 02139
}

(Manuscript received 3 August 1982, in final form 13 March 1983)

\begin{abstract}
We describe a new numerical model designed to study the interactions between hydrodynamics and thermodynamics in the upper ocean. The model incorporates both primitive equation dynamics and a parameterization of mixed layer physics. There is a consistent treatment of mixed layer structure for all physical processes.

In order to study interplay between dynamics and mixed layer physics in the equatorial ocean, we carried out a series of numerical experiments with simple patterns of wind stress and surface heating. In some cases stratification and/or mixed layer physics were suppressed. On the basis of these experiments we reached the following conclusions:

The vertical circulation at the equator is so vigorous that surface heating is essential if stratification is to be maintained for periods longer than a few months. Without stratification to inhibit mixed layer deepening momentum will be mixed uniformly to the main thermocline and the equatorial undercurrent will disappear.

Vertical transfers of momentum due to vertical advection and mixed layer entrainment are essential features of equatorial dynamics. These processes influence currents, SST and upwelling rates more than changes in sea surface elevation. Consequently, the overall mass field adjustments of equatorial oceans are more nearly linear than are the currents or SST variations.

The connection between changes in SST and dynamical quantities such as sea surface topography need not be straightforward. For example, increased upwelling will make the mixed layer shallower but will not reduce SST unless it induces increased entrainment of colder water. The influences of upwelling and downwelling on SST are highly asymmetric so that the influence of perturbations cannot be predicted without considering the mean vertical velocity.

The asymmetry in the interaction between vertical velocity and mixed layer physics can result in the formation of surface fronts. On the upwelling side of a $w=0$ line the surface layer is cold and shallow while on the downwelling side it is warm and deep. Differential advection creates a temperature discontinuity at the depth discontinuity. It is suggested that the Galapagos Front has this character.
\end{abstract}

\section{Introduction}

Over much of the world's oceans the sea surface temperature (SST) response to atmospheric forcing is determined principally by local thermodynamic processes and turbulent mixing processes. The two taken together constitute the physics of the mixed layer and one-dimensional models have often successfully simulated the evolution of SST at various ocean weather ships (e.g., Denman and Miyake, 1973; Thompson, 1976). However, in many instances ocean dynamics exert an important influence on SST; notably under severe storms, in western boundary currents, in coastal upwelling zones, and along the equator. A disproportionate share of the heat exchange with the atmosphere seems to result from these situations where dynamics drives the ocean away from thermal equilibrium. In fact, it is the premise of such studies as Busalacchi and O'Brien
(1981) that in El Niño events SST anomalies are so strongly divorced from 1-dimensional physics that it is more advantageous to study the dynamics of a single-layer reduced-gravity model.

Of course, the procedure of relating SST changes to thickness changes in the single-layer model is naive, and SST and depth of a reduced-gravity layer need not be at all correlated. But in fact, the results of Busalacchi and O'Brien seem quite plausible, and are corroborated to some extent by the work of Philander and Pacanowski (1980) and Semtner and Holland (1980), with full ocean general circulation models. The reason that the reduced-gravity models appear successful is that there does tend to be a correlation between upwelling and cooling of the surface waters. As will be seen here, however, this correlation is not exact, and is modified by many interesting and spatially varying phenomena, which can delay the SST response or mask it entirely. 
To begin to get an appreciation for the way in which the SST behaves in the presence of upwelling and other ocean dynamics, we have returned to the structure and physics associated with the turbulent well-mixed surface layer, but with the addition of primitive equation ocean dynamics to evaluate the flow in the upper ocean. We have constructed a model which treats the upper ocean as two layers which lie atop an infinitely deep abyss. The top layer is viewed as the turbulent, well-mixed layer with vertically uniform temperature and currents. The second layer is linearly stratified, and represents the remainder of the ocean above the main thermocline. It is the layer from which water is entrained into the mixed layer, so that prediction of its thermal structure is a necessary part of the attempt to accurately simulate the change in SST by entrainment. Entrainment is predicted by Kraus-Turner (1967) energetics, as modified by many subsequent investigators.

It will be necessary throughout this paper to make a clear distinction between the terms "upwelling" and "entrainment." The surface describing the base of the mixed layer is not a material surface, and therefore allows a mass flux across it. This flux is defined as entrainment (if upwards) or detrainment. But since this surface is not at a constant depth in space nor time, the entrainment is not a vertical velocity. Vertical motion of water relative to a geo-centric coordinate system is termed upwelling (or downwelling). With the mixed layer treated as vertically homogeneous and ignoring horizontal or surface fluxes, only entrainment (not upwelling nor detrainment) changes SST. Upwelling can make entrainment far more likely or effective, but until colder water enters the turbulent surface layer through entrainment, it can have no influence on the SST.

It is precisely this distinction between entrainment and upwelling which will lead to different conclusions than those arrived at by studying reduced-gravity models such as Cane's (1979) or Busalacchi and O'Brien's (1981). There is a difference between these two models which applies to this study as well: there is a real distinction to be made between models which have the stress uniformly distributed over the layer, as in Busalacchi and O'Brien, and those which distribute the stress over only a portion of the layer (Cane). In the present model the primitive equations are used to predict the velocities in each of the two layers, and the stress is applied only to the well-mixed layer. If the two layers have identical buoyancies, the model is quite similar to Cane's.

The distinguishing feature of our model is the treatment of the mixed layer as a layer for both dynamics and thermodynamics. This representation is usual in one dimensional models of mixed layer physics, but it has been less often used in models that include dynamical processes as well. Typically, models that include both dynamic and thermodynamic processes have been level models that either take no particular account of the mixed layer or simulate its physics simply by an enhanced eddy viscosity. (At best, the viscosity depends on the Richardson number, e.g., Pacanowski and Philander, 1981). Recently, a number of models have combined slab mixed layer physics with a level model for dynamics (Adamec et al., 1981). Briefly, quantities are mixed from the surface down to a mixed layer depth $h_{m}$ determined by the parameterization of mixed layer physics. The depth $h_{m}$ usually will fall in between two model levels, forcing a jump in properties within a level. The dynamics continue to treat the level as a single unit.

There are two objections to such a scheme. First, it is inefficient. Either the near surface resolution is inadequate or there will usually be a number of levels within the mixed layer-each level repeats the same values of the variables and the same calculation of advective terms. Second, such a procedure is inherently inconsistent: the parameterization of mixed layer physics requires a discontinuity at $h_{m}$ while the numerical treatment of the dynamics implicitly assumes continuous variations in the vertical. In view of the coarseness of the vertical grid, the changes at the base of the mixed layer are properly modeled as discontinuous. We use this representation consistently for both dynamics and thermodynamics.

This model structure is similar to that in Price (1981). Its principal difference lies in the treatment of the entrainment equation and the thermal structure below the mixed layer. Specifically, there is no provision for detrainment allowed in Price's model. While this was sufficient for a study of the hurricane response, it would prove unacceptable in the longterm integrations envisioned here. Pollard (1982) has constructed a model along similar lines, except that each layer has a temporally and spatially constant density, uninfluenced by the water temperature. Thus there is no feedback between the thermal processes and dynamics.

Given the present understanding of mixed layer physics, the representation we use for the mixed layer seems optimal and will be maintained in all envisioned versions of the model. The additional simplifications of vertical structure specific to the present version restrict the class of phenomena that the model is suited to investigate. We use the simplest vertical structure that will allow us to investigate the interaction of dynamics and thermodynamics in the upper portion of the tropical ocean. Not surprisingly our model turns out to be an extension of the model of Cane (1979) to include thermal processes. That model anticipated the essential results of more elaborate numerical models (e.g., compare Cane with Philander and Pacanowski, 1980).

The model is set forth in Sections 2 and 3. To examine the implications of these modeling choices and to initiate studies of equatorial SST changes, we 
have run the model in an equatorial box and examined the response to the sudden onset of easterly and southerly winds, reported in Section 4. Some of the implications of our results are considered in Section 5 .

\section{Model description-Physics}

\section{a. Generalized coordinates}

The model solves reduced-gravity primitive equations for the upper few hundred meters of the ocean. It proves convenient to replace the usual vertical coordinate $z$ by a generalized vertical coordinate- $s$ $=s(\lambda, \theta, z, t)$. We define

$$
h(\lambda, \theta, s, t)=\partial z / \partial s,
$$

with $z=0$ at $s=0$.

The vertical motion relative to $z$ is replaced by that relative to $s$ : let $w_{e}$ be the volume flux per unit area across surfaces of constant $s$. Then

$$
w_{e}=h d s / d t=w-\partial z / \partial t+\mathbf{u} \cdot \nabla z
$$

where it is understood that all temporal and horizontal spatial derivatives are to be taken with $s$ held constant, and where $w$ is the vertical motion in the $z$-system.

After making the Boussinesq approximation, the governing equations for evolution of momentum, heat and mass are (Kasahara, 1974):

$$
\begin{gathered}
\frac{\partial(h \mathbf{V})}{\partial t}+\nabla \cdot(\mathbf{V} h \mathbf{V})+\frac{\partial\left(w_{e} \mathbf{V}\right)}{\partial s}-[f+u /(a \tan \theta)] \mathbf{k} \\
\times h \mathbf{V}=-h\{\nabla p+b \nabla z\}+\frac{\partial \tau}{\partial s}+h F_{H}(\mathbf{V}), \\
\frac{\partial(h T)}{\partial t}+\nabla \cdot(\mathbf{V} h T)+\frac{\partial\left(w_{e} T\right)}{\partial s} \\
=\frac{-\partial Q}{\partial s}-\frac{\partial D_{v}}{\partial s}+h D_{H}(T), \\
\frac{\partial h}{\partial t}+\nabla \cdot(h \mathbf{V})+\frac{\partial w_{e}}{\partial s}=0,
\end{gathered}
$$

where $F_{H}(\mathbf{V})$ is a horizontal mixing of momentum, $D_{H}(T)$ is a horizontal diffusion operator, $Q$ is an upward flux of heat (divided by $\rho c_{p}$ ), and $D_{v}$ is an upward diffusive flux. The hydrostatic relation is

$$
\frac{\partial P}{\partial s}=b h,
$$

where $b$ is the buoyancy, calculated with only a linear thermal expansion:

$$
b=b(T)=g \alpha\left(T-T_{r}\right),
$$

with $\alpha$ constant and $T_{r}$ a reference temperature. It remains to specify an equation for $w_{e}$; this process is discussed below in Section 2.4.

\section{b. Vertical structure}

The vertical structure of the model is chosen to efficiently represent the important features of the upper ocean-the surface mixed layer and seasonal thermocline. Two layers are retained: a surface mixed layer, homogeneous in temperature $\left(T_{1}\right)$ and velocity $\left(V_{1}\right)$ with a thickness $h_{1}$. The second layer, of thickness $h_{2}$, has a velocity $V_{2}$, and is linearly stratified in the vertical, with a depth-averaged temperature $T_{2}$ and a temperature $T_{b}$ at its base, as shown in Fig. 1 . The deep water beneath these layers has temperature $T_{r}$, sustains no baroclinic pressure gradients and is motionless. This simplification to a reduced gravity model is a common one (cf. Cane, 1979, and references therein) and is appropriate for studies of short timescale (several years or less) variations in the upper ocean.

As in other slab models of mixed layer physics (e.g., Niiler and Kraus, 1977) we assume:

1) The influence of the turbulent stresses generated within the mixed layer is confined to the mixed layer.

2) Temperature, salinity and buoyancy are vertically uniform within the mixed layer.

3) Horizontal velocity is vertically uniform within the mixed layer.

4) The changes in temperature and velocity at the base of the mixed layer take place in such a narrow region in the vertical that they may be modeled as discontinuous.

Assumptions (1), (2) and (4) are strongly supported by observations. While there is some evidence of significant velocity shear within the mixed layer (e.g., Davis et al., 1981; Halpern, 1980), this shear is typically small compared to the velocity difference be-

MODEL STRUCTURE

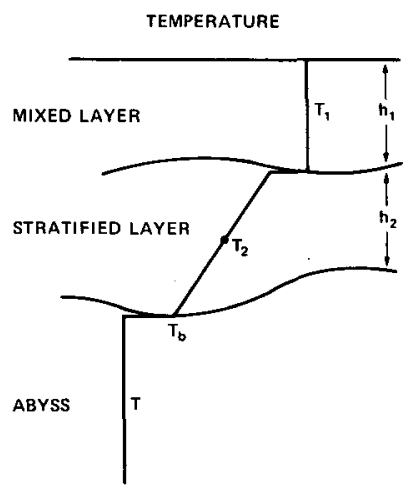

VELOCITY

FIG. 1. Vertical structure of temperature and velocity assumed in the model equations. $T_{k}, h_{k}$ and $\mathbf{V}_{k}$ vary with time, latitude and longitude. 
tween the mixed layer and the ocean immediately below it, making assumption (3) reasonable. The model treats the vertical structure consistently, using the same assumptions for prediction of horizontal dynamics, entrainment prediction and for evaluating vertical fluxes of heat and momentum.

The coordinate surfaces are chosen so that

$$
\left.\begin{array}{c}
z(s=0)=0 \\
z(s=-1)=-h_{1} \\
z(s=-2)=-h_{1}-h_{2}
\end{array}\right\},
$$

where $h_{1}$ is the depth of the mixed layer and $h_{2}$ is the thickness of the second layer. The scale factor $h$ is taken to be constant within a layer (i.e., $h(s)=h_{k}$ for $-(k+1)<s<-k)$, and is therefore synonymous with layer thickness.

Since the ocean surface and the main thermocline are material surfaces $w_{e}(s=0)=w_{e}(s=-2)=0$; $w_{e}(s=-1)$ is the rate of entrainment into the mixed layer and therefore is determined by the parameterization we choose for mixed layer physics. Hereafter when we write $w_{e}$ without an explicit argument it will be understood to be $w_{e}(-1)$.

To aid in the description of the second layer stratification, we define $\Gamma$ :

where

$$
\Gamma=\frac{\partial T}{\partial s}=h \frac{\partial T}{\partial z}=T_{e}-T_{b}
$$

$$
\begin{aligned}
& T_{e}=\lim _{s t-1} T(s)=T_{2}+\frac{\Gamma}{2}, \\
& T_{b}=\lim _{s t-2} T(s)=T_{2}-\frac{\Gamma}{2} .
\end{aligned}
$$

The continuous description of temperature within the second layer is thus

$$
\begin{gathered}
T=T_{2}(\lambda, \theta)+\left(s+\frac{3}{2}\right) \Gamma(\lambda, \theta) \\
-2<s<-1
\end{gathered}
$$

The representation of vertical structure in a two layer model is necessarily crude. The extra degree of freedom $(\Gamma)$ for the second layer temperature permits a much better approximation to the stratification at the base of the mixed layer, a critical parameter for entrainment.

\section{c. Model equations}

Equations for $\mathrm{V}_{k}, h_{k}, T_{k},(k=1,2)$ are derived from (2.5), (2.3) and (2.4) by integrating each equation over layer $k$ :

$$
\text { (i.e., } \left.\int_{-k}^{-k+1}[] d s\right) \text {. }
$$

The results are

$$
\begin{gathered}
\frac{\partial\left(h_{1} \mathbf{V}_{1}\right)}{\partial t}+\nabla \cdot\left(h_{1} \mathbf{V}_{1} \mathbf{V}_{1}\right)+\left[f+\frac{u_{1}}{a \tan \theta}\right] \mathbf{k} \times h_{1} \mathbf{V}_{1} \\
=\tau_{0}-\tau_{1}-h_{1}\left[\nabla\left(b_{1} h_{1}+b_{2} h_{2}\right)-h_{1} \nabla \frac{b_{1}}{2}\right] \\
+h_{1} F_{H}\left(\mathbf{V}_{1}\right)+w_{e}\left\{H\left(w_{e}\right) \mathbf{V}_{2}+H\left(-w_{e}\right) \mathbf{V}_{1}\right\} \\
\frac{\partial\left(h_{1}\right)}{\partial t}+\nabla \cdot\left(h_{1} \mathbf{V}_{1}\right)=w_{e}
\end{gathered}
$$$$
\frac{\partial\left(h_{1} T_{1}\right)}{\partial t}+\nabla \cdot\left(h_{1} \mathbf{V}_{1} T_{1}\right)=Q_{1}-D_{1}+h_{1} D_{H}\left(T_{1}\right)
$$$$
+w_{e}\left\{H\left(w_{e}\right) T_{e}+H\left(-w_{e}\right) T_{1}\right\},
$$$$
\frac{\partial\left(h_{2} \mathbf{V}_{2}\right)}{\partial t}+\nabla \cdot\left(h_{2} \mathbf{V}_{2} \mathbf{V}_{2}\right)+\left[f+\frac{u_{2}}{a \tan \theta}\right] \mathbf{k} \times h_{2} \mathbf{V}_{2}
$$$$
=\tau_{1}-\tau_{2}-h_{2}\left[\nabla\left(\frac{b_{2} h_{2}}{2}\right)+b_{2} \nabla\left(h_{1}+\frac{h_{2}}{2}\right)\right.
$$$$
\left.-\frac{\Gamma_{b} \nabla h_{2}}{6}-\frac{\nabla \Gamma_{b} h_{2}}{12}\right]+h_{2} F_{H}\left(\mathbf{V}_{2}\right)
$$$$
-w_{e}\left\{H\left(w_{e}\right) \mathbf{V}_{2}+H\left(-w_{e}\right) \mathbf{V}_{1}\right\} \text {, }
$$$$
\frac{\partial\left(h_{2}\right)}{\partial t}+\nabla \cdot\left(h_{2} \mathbf{V}_{2}\right)=-w_{e},
$$$$
\frac{\partial\left(h_{2} T_{2}\right)}{\partial t}+\nabla \cdot\left(h_{2} \mathbf{V}_{2} T_{2}\right)=Q_{2}+D_{1}+h_{2} D_{H}\left(T_{2}\right)
$$$$
-w_{e}\left\{H\left(w_{e}\right) T_{e}+H\left(-w_{e}\right) T_{1}\right\} .
$$

$H$ is the Heaviside step function $H(x)=1$ if $x>0$, $H(x)=0$ if $x<0 ; Q_{k}$ is the diabatic heating in layer $k ; \tau_{k}\left(D_{k}\right)$ is the stress (diffusion) between layer $k$ and layer $k+1$ with $\tau_{0}$ the surface wind stress. $\Gamma_{b}$ is the linear gradient in buoyancy in the second layer $\left(\Gamma_{b}\right.$ $=g \alpha \Gamma)$. The terms in braces reflect the discontinuity at the base of the mixed layer; in (2.11) for example, this term shows that when the mixed layer is deepening $\left(w_{e}>0\right)$ it acquires fluid with momentum $\mathbf{V}_{2}$; when it becomes shallower $\left(w_{e}<0\right)$ it loses fluid with momentum $V_{1}$. The model thus uses upstream differencing in the vertical. According to (2.11)-(2.16) mixed layer processes $\left(w_{e} \neq 0\right)$ conserve momentum, mass and heat (but not energy; see below).

The temperature at the base of the second layer is predicted from (2.4) evaluated at $\lim s \downarrow-2$ :

$$
\begin{aligned}
\partial\left(h_{2} T_{b}\right) / \partial t+\nabla \cdot\left(h_{2} \mathbf{V}_{2} T_{b}\right) & +w_{e} T_{b} \\
& =h_{2} D_{H}\left(T_{b}\right)+D_{b} .
\end{aligned}
$$

In (2.17) $T_{b}$ is uninfluenced by mixed layer processes, except during some detrainment episodes (the role of $w_{e}$ in (2.17) is to balance $\left.\partial h_{2} / \partial t+\nabla \cdot\left[h_{2} \mathbf{V}_{2}\right]\right)$. The linear stratification does not allow enough degrees of freedom to represent the complex thermal structure left behind in the second layer after detrainment. If 
the adjustment to a linear profile (cf. 2.10 ) results in static instability $\left(T_{e}>T_{1}\right)$ then $T_{b}$ is increased by vertical diffusion (term $D_{b}$ ) just enough to restore stability. (Otherwise, $D_{b}=0$ ).

Our treatment of entrainment and detrainment does not falsify the mixed layer depth or temperature. It conserves heat and momentum but not energy. The energy changes are

for

$$
-\left|w_{e}\right|\left\{\frac{\left(\mathbf{V}_{1}-\mathbf{V}_{2}\right)^{2}}{2}+h_{1} \frac{\left(b_{1}-b_{e}\right)}{2}\right\},
$$

for

$$
\begin{gathered}
w_{e}>0, \\
-\mid w_{e}\left\{\frac{\left(\mathbf{V}_{1}-\mathbf{V}_{2}\right)^{2}}{2}+h_{1} \frac{\left(b_{1}-b_{e}\right)}{3}\right\},
\end{gathered}
$$

$$
w_{e}<0 \text {. }
$$

In the case of entrainment $\left(w_{e}>0\right)$ there is a kinetic energy loss related to the velocity shear at the base of the mixed layer and a potential energy gain as more buoyant fluid is mixed downward. These changes are fully accounted for in the energy balance equations used to predict entrainment (cf. Eq. 2.21 below). In fact, a number of mixed layer parameterizations are based on the idea that the potential energy increase associated with entrainment results from the turbulent motions produced by the conversion of mean flow kinetic energy in the shear zone at the base of the mixed layer (e.g., Pollard et al., 1973). In the case of detrainment $\left(w_{e}<0\right)$ the energy losses result from the artificial mixing of buoyancy and momentum required to restore linear stratification in the second layer and to keep but a single velocity variable for the second layer. Inclusion of the traditional form of vertical diffusion would have the same effect.

\section{d. Entrainment rate}

In order to close the set of equations we must determine the rate of entrainment into the surface mixed layer. Three possible choices are allowed:

1) A conventional layer model:

$$
w_{e}=0
$$

2) A constant depth surface mixed layer:

$$
w_{e}=\nabla \cdot\left(h_{1} \mathbf{V}_{1}\right)
$$

so that $\partial h_{1} / \partial t=0$. With this choice the model may be stratified as described above or unstratified ( $T_{1}$ $=T_{2} ; \Gamma=0$ ).

3) A parameterization of the physics of the windmixed layer based on the bulk turbulent kinetic energy budget. This is essentially the Kraus and Turner (1967) model, with modifications suggested by a number of subsequent investigators (see Niiler and Kraus, 1977 and Garwood 1979 for reviews). In using this parameterization we are implicitly assuming that it is not altered by interaction with large scale dynamics.

Niiler and Kraus give a detailed discussion of energy budget models for mixed layer deepening. Their equation (10.30) is applied here, with the addition of a background dissipation term (Kim, 1976):

$$
\begin{aligned}
& w_{e} H\left(w_{e}\right)\left\{h_{1}\left(b_{1}-b_{e}\right)+q^{2}-m_{e}|\Delta V|^{2}\right\} \\
& \quad=2 m_{s} u^{* 3}-2 \epsilon_{0} h_{1}+h_{1} B_{0}\left[1-m_{b} H\left(B_{0}\right)\right]+B_{1}(h) .
\end{aligned}
$$

Here $u^{*}$ is the surface friction velocity, $B_{0}$ the surface buoyancy flux and $B_{1}$ is the rate of potential energy generation due to penetrating radiation. Here $q^{2}$ is the turbulence energy level in the mixed layer, and $\Delta V^{2}$ is the velocity shear across the interface. The parameters $m_{e}, m_{s}, m_{b}$ arise through parameterization of the total dissipation as proportional to production of turbulent energy by shear instabilities, surface processes, and convection. Reasonable values for these terms and a discussion of their importance may be found in Niiler and Kraus (1977) and Garwood (1979). Different choices for the parameters allow us to simulate many different published mixed layer models.

We include a depth dependent background dissipation term $\epsilon_{0} h_{1}$, which limits mixed layer growth. Some investigators have used a term with a stronger depth dependence, e.g., exponential (see Garwood). We can achieve the same effect by imposing a condition

$$
h_{1}<h_{\max } \text {, }
$$

(i.e., for $h_{1}>h_{\max }, \epsilon_{0} \rightarrow \infty$ ), though the need to do so does not arise in the present study. It is known that the effects of the penetrating radiation and of gusty winds are important in preventing the mixed layer from becoming extremely shallow during strong heating regimes (e.g., Woods, 1980). If we do not wish to explicitly simulate such phenomena we parameterize their effect by requiring that

$$
h_{1} \geqslant h_{\min } \text {. }
$$

In the present study we do not take account of the depth-varying influence of penetrating radiation except through this device.

\section{e. Surface fluxes, sidewall boundary conditions and turbulent mixing}

The ocean is driven by the surface fluxes of momentum, heat and turbulent energy (wind stirring, wave breaking, etc.). We distinguish the wind stress (divided by density) $(\tau)$ from the square of the friction velocity $\left(u^{* 2}\right)$. The former is a vector quantity that drives the mean currents and often may be adequately 
represented by long-time averages. The friction velocity is used to parameterize the generation of turbulent energy $\left(u^{* 3}\right)$ and requires a different average of a scalar quantity.

\section{1) SIDEWALl BOUNDARY CONDITIONS}

The model code allows for either free-slip or noslip boundary conditions at the sidewalls. In the present study we use the no slip condition $V=0$ along the walls. As a thermal condition the model allows the specification of either the heat flux (effectively $\partial T / \partial n$ ) or the temperature $T$. We use the no heat flux condition

$$
\partial T / \partial x=0
$$

at the meridional boundaries of the model basin and specify $T$ at the zonal walls to compensate for the fact that the model does not include the mid-latitude portion of the oceans.

\section{2) TURBulent MiXING}

The principal vertical transfers due to small scale turbulence are those associated with mixed layer entrainment. The model also allows for explicit friction and diffusion between layers 1 and 2 of the form

$$
\begin{aligned}
\tau_{1} & =K_{m 1}\left(\mathbf{V}_{1}-\mathbf{V}_{2}\right), \\
D_{1} & =K_{T 1}\left(T_{1}-T_{2}\right) .
\end{aligned}
$$

We will take $K_{m 1}=K_{T 1}=0$ so that only entrainment processes act as friction. The model code also allows for turbulent transfers between the second layer and the ocean below, but these are also taken to be zero in the present study.

Without some horizontal eddy diffusion the boundary conditions on $V$ and $T$ are improperly posed. While there is certainly some horizontal viscosity in the oceans, its amplitude and form are notoriously uncertain. We take the point of view that the Laplacian form of mixing length theory is reasonable at the boundaries where there is a significant horizontal component to cross-isopycnal mixing, but that there is no physical reason to impose any horizontal mixing in the ocean interior. In our model horizontal viscosity is implemented as a part of a filtering operator that is otherwise properly regarded as part of the finite difference scheme. This is discussed further in Section 3c.

\section{Numerical methods}

Our basic time differencing scheme is the "splitting up" method (Marchuk, 1974): for an equation of the form $\partial u / \partial t=F(u)$ the operator on the right hand side may be split into parts (i.e., $F=A+B+C$ $+\cdots)$ with each part treated differently. We split the governing equations (2.11)-(2.17) together with one of (2.19)-(2.21) into three parts: adiabatic, in- viscid dynamics together with explicit vertical diffusion; mixed layer physics and determination of the entrainment rate, including (if needed) diabatic heating; horizontal turbulent mixing and smoothing.

\section{a. Adiabatic inviscid dynamics}

By adiabatic, inviscid dynamics we mean Eqs. (2.11)-(2.17) with $w_{e}$, all turbulent mixing terms (except the wind stress, $\tau_{0}$ ) and all diabatic heating terms neglected, leaving horizontal flux, Coriolis, and pressure force terms in addition to the time rate of change. The time differencing procedure used for this portion of the calculation is the $N$-cycle scheme of Lorenz (1971) with $N=4$ (also see Israeli and Gottlieb, 1974, and Cane, 1979).

The model equations are treated in energy conserving flux form on a finite difference latitude-longitude grid. The grid layout is chosen as in Arakawa's (1972) scheme B: temperature, layer depth and entrainment velocity are defined at the center of a grid box; horizontal velocities are defined at the four corners. Boundary points coincide with velocity points, making it easier to set boundary conditions. The grid may have variable resolution; the grid stretching procedure is as described in Cane (1979). Grid configuration $B$ is superior to other schemes when the grid spacing is not small compared to the radius of deformation.

\section{b. Entrainment and mixed layer physics}

The second step in the splitting-up scheme is the determination of the entrainment rate $w_{e}$ and its consequences. The results of a complete cycle of the inviscid adiabatic step are used as initial conditions. In the layer model configuration Eq. (2.19) applies, $w_{e}$ $=0$ and this step is unnecessary. With the constant level formulation integration of the continuity equation (2.12) in the previous step will have changed the surface layer depth from its constant value $h_{\mathrm{fix}}$ to a new value $h_{1}^{0}$. Therefore this step must impose a depth change

$$
d h=h_{\mathrm{fix}}-h_{1}^{0}
$$

to restore the surface layer depth to the value $h_{\mathrm{fix}}$. Note that with $w_{e} \Delta t=d h(3.1)$ is consistent with (2.12) and (2.20).

The mixed layer equations are solved by integrating the entrainment equation over the timestep, with the results of the inviscid adiabatic step as initial conditions. The equation is integrated analytically. This is done most easily if the equation (2.23) is recast in energy balance form. Neglecting penetrating radiation and the change in turbulent kinetic energy,

$$
d\left(P E+m_{e} M K E\right) / d t=m_{s} u_{*}^{3}-\epsilon_{0} h,
$$

where $P E$ is the mean potential energy and $M K E$ is the mean kinetic energy. We will assume that the 
forcing $\left(u_{*}\right.$ and $\left.Q\right)$ is constant over the timestep, and will approximate

$$
\int_{t_{0}}^{t_{0}+\Delta t} \epsilon_{0} h d t \approx \epsilon_{0}\left(h_{1}+d h\right) \Delta t
$$

where $h_{1}$ is the mixed layer depth at the beginning of the timestep $\left(t_{0}\right)$ and $h_{1}+d h$ is the depth at the end of the timestep $\left(t_{0}+\Delta t\right)$. Hence

$$
d h=\int_{t_{0}}^{t_{0}+\Delta t} w_{e} d t .
$$

We may now write (3.2) in the form $F(d h)=0$. If the mixed layer is deepening

$$
\begin{aligned}
F(d h)=-[ & \left.2 m_{s} u^{* 3}-2 \epsilon_{0} h_{1}-B_{0} h_{1}\right] \Delta t \\
+\left[B_{0} \Delta t+\right. & \left.h_{1} \delta b+2 \epsilon_{0} \Delta t\right] d h+\left[N^{2} \frac{h_{1}}{2}\right] d h^{2} \\
+ & {\left[\frac{N^{2}}{6}\right] d h^{3}-m_{e}(\Delta V)^{2} \frac{h_{1} d h}{\left(h_{1}+d h\right)}, }
\end{aligned}
$$

where $\delta b=b_{1}-b_{e}$ is the jump in buoyancy at the base of the mixed layer at the $t=t_{0}, \Delta V$ is the jump in speed, $B_{0}=g \alpha Q$, and $N^{2}=g \alpha \Gamma / h_{2}$ is the buoyancy gradient in the second layer. Eq. (3.3) is a quartic polynomial in $d h$ and is easily solved; moreover, it is usually dominated by the lowest order terms.

Eq. (3.3) applies only if there is a positive root less than $h_{2}$. For a shallowing layer, we treat the problem as one of the formation of a new mixed layer shallower than the old one. We take $h_{1}, \delta b, \Delta V$ and $N^{2}$ to be zero, in (3.3); disregarding changes in turbulent kinetic energy:

$$
F\left(h_{d}\right)=-2 m_{s} u_{*}^{3}+\left(B_{0}+2 \epsilon_{0}\right) h_{d}=0,
$$

where, with $d h$ again the change in layer depth from its value $h_{1}$ at $t=t_{0}$, we have

$$
d h=h_{1}-h_{d} .
$$

When the model is run with the top layer controlled either by the fixed depth or mixed-layer energetics, there is a mass flux between layers 1 and 2 which will carry heat and momentum as reflected in the $w_{e}$ terms in Eqs. (2.11)-(2.17). With entrainment, the fluid in the region between $z=h_{1}$ and $z=h_{1}$ $+d h$ was in layer 2 at $t=t_{0}$, and is entrained into layer 1 at $t=t_{0}+\Delta t$; its properties should be transferred accordingly. For example, for momentum:

$$
\begin{aligned}
& \mathbf{V}^{\prime} h_{1}^{\prime}(\Delta t)=\mathbf{V} h_{1}^{0}+d h \mathbf{V}_{2}^{0}, \\
& \mathbf{V}^{\prime} h_{2}^{\prime}(\Delta t)=\mathbf{V} h_{2}^{0}-d h \mathbf{V}_{2}^{0},
\end{aligned}
$$

where $d h$ is determined from the appropriate one of (3.1) or (3.3) and superscripts zero and prime refer to the values before and after the entrainment. With a detraining surface layer $[d h<0$ determined from
(3.1) or (3.5)] the fluid between $z=h_{1}-|d h|$ and $z$ $=h_{1}$ is "left behind" in layer 2 :

$$
\begin{aligned}
& h_{1}^{\prime} \mathbf{V}_{1}^{\prime}=h_{1}^{0} \mathbf{V}_{1}^{0}-|d h| \mathbf{V}_{1}^{0}, \\
& h_{2}^{\prime} \mathbf{V}_{2}^{\prime}=h_{2}^{0} \mathbf{V}_{2}^{0}+|d h| \mathbf{V}_{1}^{0} .
\end{aligned}
$$

\section{c. Smoothing and filtering}

As discussed in Section 2e, we do not rely on explicit horizontal diffusion of heat and momentum to control small scale numerical noise. Instead we apply a high-order Shapiro (1970) filter to the variables. This provides for highly scaled selective damping, eliminating waves with lengths of $2 \Delta x$, while leaving longer waves quite unchanged. The filter is applied in each of the two coordinate directions sequentially to give the 2-dimensional version, and is applied to $h, T, u \bar{h}^{x y}$ and $v \bar{h}^{x y}$. In lieu of specifying very high order boundary conditions, the order of the filter is reduced at the walls, starting with a second-order filter applied at the grid point adjacent to the wall, and increasing the order by 2 at each successive point inwards, until the ultimate order is reached. In the present calculation, we use an eighth-order filter in the interior.

Mixing is greatest around the boundaries, as a consequence of the reduced order there. This choice was favored over the alternative of specifying high order derivatives at the walls because of the difficulty in establishing a physical basis for higher order closure and because of the reasonableness of having larger cross-isopycnal mixing due to continental topography, tidal mixing, etc. The filter is viewed as a necessary stabilizing feature of the numerical scheme, rather than a parameterization of explicit physical processes, since the damping of the smallest scale noise is much larger than that indicated by physical arguments (see Kalnay-Rivas and Hoitsma, 1980).

\section{Model response}

An important region where the interplay between ocean dynamics and mixed layer behavior can be great is the tropical ocean. We have run the model in 5 cases designed to explore the nature of this interaction and to identify the dominant features of the upper ocean response to wind driving:

- In Case 1 , we fixed the mixed layer depth and eliminated all the internal temperature gradients, thus mimicking Cane (1979). This case with easterly winds serves as a benchmark and a verification of the model's dynamics.

- Case 2 is the case of greatest interest. Easterly wind drove the model with the full mixed layer physics included. To examine the role of variable mixed layer depth, a contrasting run (Case 3 ) was made with the mixed layer depth fixed. Case 3 differs from Case 1 in that the thermal field was initially stratified, and the SST field evolves in time. 
- Cases 4 and 5 illustrate the response to meridional winds as typically found in the eastern Atlantic and Pacific Oceans. They illustrate the non-linear effects involved in determining SST by comparing the response with southerly (Case 4) and southeasterly (Case 5) winds.

In all the cases the model was run in a $30^{\circ} \times 30^{\circ}$ box centered on the equator; grid spacing was $0.5^{\circ}$ in longitude, and stretched in the meridional direction giving $22 \mathrm{~km}$ resolution at the equator. Uniform winds were impulsively applied at time $t=0$, and continued for the duration of the runs. For those cases with the mixed layer physics included $(2,4,5)$, the mixed-layer equations were treated with only the simplest terms retained: except for a dissipation constant of $\epsilon_{0}=7.5 \times 10^{-9} \mathrm{~m}^{2} \mathrm{~s}^{-3}$, and a surface production coefficient $m_{s}=1.25$, the other mixed layer constants $\left(m_{b}, m_{e}, q^{2}\right)$ were zero; $g \alpha=2 \times 10^{-3} \cdot \mathrm{m} \mathrm{s}^{-2} \mathrm{~K}^{-1}$. In this case study $u_{*}$ was specified at $0.864 \mathrm{~cm} \mathrm{~s}^{-1}$, resulting in an initial diagnostic (Monin-Obukhov) mixed layer depth of about $50 \mathrm{~m}$ : in the absence of advection, upwelling or anomalous heating, the dissipation, production and heating terms will balance at the diagnostic depth. Displacements from this diagnostic depth can only occur due to vertical motion and (to a lesser extent) horizontal advection or changes in surface flux caused by changes in mixed layer temperature.

The initial conditions consisted of an ocean at rest. In all cases the depth and temperature fields were horizontally uniform, with $h_{1}+h_{2}=200 \mathrm{~m}$. Except for Cases 1 and 3 , which had $h_{1}=25 \mathrm{~m}$, the initial mixed layer depth was $50 \mathrm{~m}$. For cases 2 through 5 , $T_{1}=28.0^{\circ} \mathrm{C}, T_{2}=19.5^{\circ} \mathrm{C}, \Gamma=10.0^{\circ} \mathrm{C}$, and $T_{r}=10.5^{\circ} \mathrm{C}$. The minimum mixed layer depth was $20 \mathrm{~m}$.

Case $1 \mathrm{had}$ the mixed layer fixed at $25 \mathrm{~m}$, and the two active layers are homogeneous in temperature $\left(T_{1}=T_{2}=28.0^{\circ} \mathrm{C}, \Gamma=0\right.$ and $\left.T_{r}=19.1^{\circ} \mathrm{C}\right)$. There is no surface heating, so the model retains its initial temperature structure throughout the run. An easterly wind stress of $0.465 \mathrm{dyn}^{-2}$ is applied at day 0 . The variables of interest are the velocity and the pycnocline height anomaly (PHA) - the displacement of the second interface from its initial depth. Fig. 2. gives the time evolution of these fields along the equator.

The model ocean response is very much like that described in Cane (1979). Briefly, the initial response consists of the excitation of a jet (Yoshida, 1959) along the equator plus Kelvin and Rossby wave fronts off the western and eastern walls. Since there is no density difference between the layers, only the first baroclinic mode exists, and the interfacial depth change and velocity fields show this single propagation speed. There is a velocity shear between the layers because only the upper layer is directly driven by
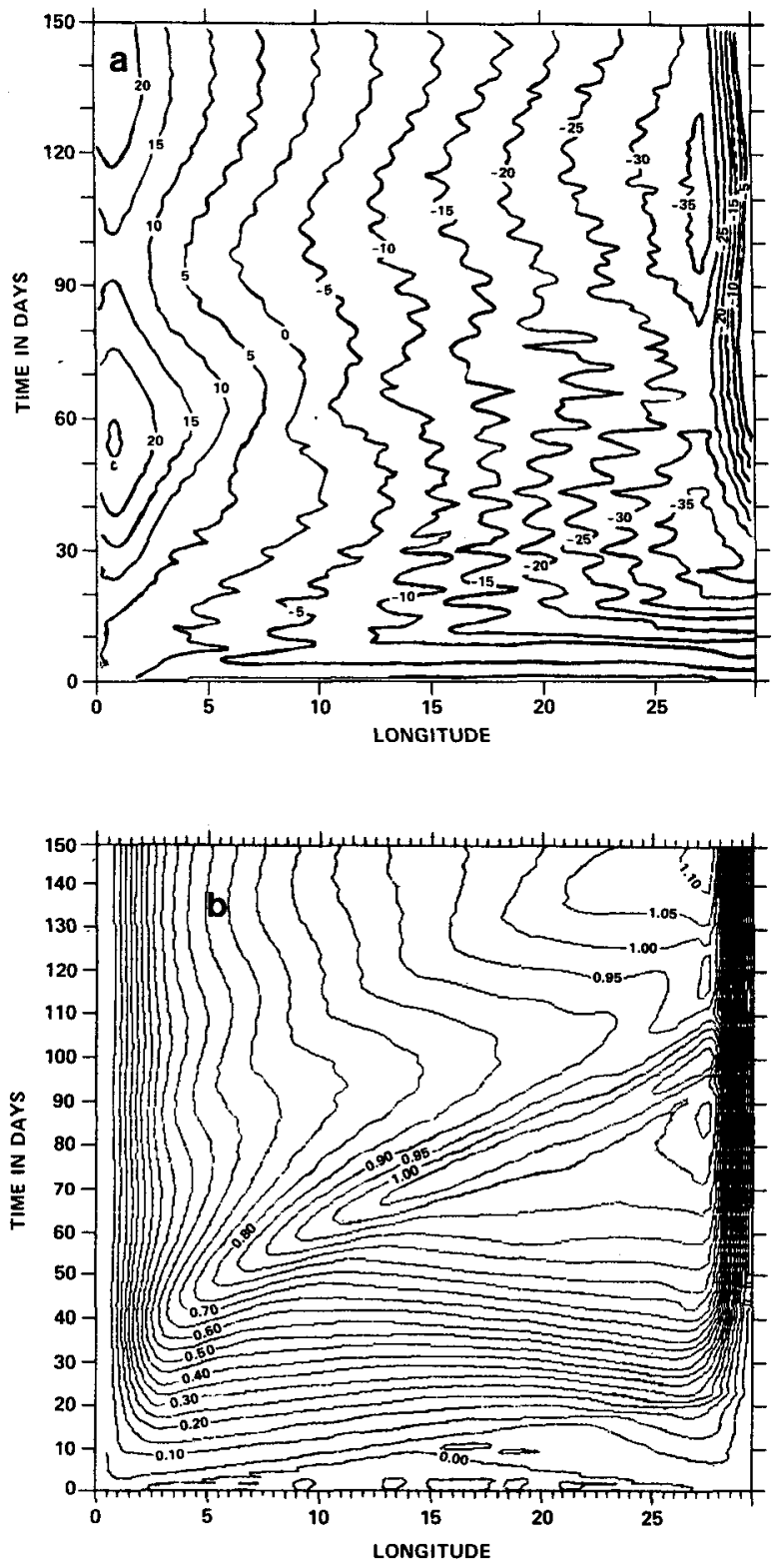

FIG. 2. Time-longitude sections of (a) pycnocline height anomaly and (b) undercurrent speed along the equator from Case 1 . The mixed layer depth is held constant at $25 \mathrm{~m}$, and the model is unstratified, as in Cane (1979b). Contour intervals are of $5 \mathrm{~m}$ and $5 \mathrm{~cm} \mathrm{~s}^{-1}$.

the wind stress. The other dynamical time scale that enters is the frictional one $\mathrm{O}(10$ days) governing the spin-up of the surface layer (see Cane 1979).

The results in Case 1 differ from those in Cane (1979) only in details. Some of the differences are attributable to differences in numerical methods, but the most significant ones appear to result from different physical assumptions about the nature of momentum exchanges between the two active layers. In the present model there is no explicit vertical diffu- 
sion: with upwelling, all momentum exchange is by surface layer entrainment [cf. Eqs. (2.11) and (2.14)]:

$$
\begin{aligned}
& \frac{h_{1} \partial \mathbf{V}_{1}}{\partial t}=-w_{e}\left(\mathbf{V}_{1}-\mathbf{V}_{2}\right)+\cdots, \\
& \frac{h_{2} \partial \mathbf{V}_{2}}{\partial t}=-w_{e}\left(\mathbf{V}_{2}-\mathbf{V}_{2}\right)+\cdots \cdot
\end{aligned}
$$

In Cane (1979) there is explicit diffusion and vertical advection is assumed to be energy conserving; in our notation

$$
\begin{aligned}
& \frac{h_{1} \partial \mathbf{V}_{1}}{\partial t}=-w\left(\mathbf{V}_{1}-\mathbf{V}_{2}\right) / 2-K\left(\mathbf{V}_{1}-\mathbf{V}_{2}\right) \\
& \frac{h_{2} \partial \mathbf{V}_{2}}{\partial t}=-w\left(\mathbf{V}_{1}-\mathbf{V}_{2}\right) / 2+K\left(\mathbf{V}_{1}-\mathbf{V}_{2}\right)
\end{aligned}
$$

where the friction coefficient $K$ can be written as $K$ $=\nu / H_{*}$ with $\nu$ a vertical eddy viscosity and $H_{*}$ a characteristic vertical scale (see Cane, 1979). The two sets of formulas (4.1) and (4.2) are equivalent if $K$ $=w / 2$ and $w=w_{e}$. This identification is one way of appreciating the equivalent frictional effect of surface layer entrainment [also, cf. (2.18)]. For Case 1, $w_{e}$ on the equator is $4-8 \mathrm{~m} \mathrm{day}^{-1}$, corresponding to $\nu$ $\approx 23-46 \mathrm{~cm}^{2} \mathrm{~s}^{-1}$ with $H_{*}=100 \mathrm{~m}$. The mean upwelling between $\pm 1.5^{\circ}$ is $2.3 \mathrm{~m} \mathrm{day}^{-1}$, or $\nu \approx 10 \mathrm{~cm}^{2}$ $\mathrm{s}^{-1}$. In Cane $\nu=15 \mathrm{~cm}^{2} \mathrm{~s}^{-1}$; hence the net upward transfer of momentum from the undercurrent is somewhat greater in the present model. This difference is most evident in the reversal of the surface flow, which is stronger in the present calculation.

\section{a. Model response with mixed layer physics included}

In Case 2 the mixed layer depth is determined by the parameterization (2.21). Again, an easterly stress of 0.465 dyn $\mathrm{cm}^{-2}$ is applied. Our first attempt was with the "simplest" case of zero surface heat flux, but we quickly discovered that because the vertical circulation at the equator is so vigorous the two layers soon become homogenized there. Once the stratification vanishes "deep convection" sets in, with the mixed layer deepening through the second layer and mixing momentum uniformly down to the thermocline. Thus, our first result with a mixed layer parameterization is that surface heating is essential for realistic modeling of equatorial dynamics. Without it the stratification cannot be maintained and hence the strong vertical shears associated with the undercurrent cannot be supported. (Pacanowski and Philander (1981) report similar results). To overcome this problem without introducing unnecessary complexity, we specify the surface heating as (cf. Haney, 1971):

$$
Q=30 \mathrm{~W} \mathrm{~m}^{-2} \mathrm{~K}^{-1}\left(T_{A}-T_{1}\right),
$$

where $T_{A}=29^{\circ} \mathrm{C}$.
The response in this case to easterly wind consists of a series of regimes in the thermal and dynamics fields. While these changes are not independent, we shall discuss the evolution of the dynamic quantities first, then examine the thermal fields.

In this case the currents along the equator evolve in a somewhat different fashion from those in Case 1 and Cane (1979). The buoyancy difference between the two layers permits a second baroclinic mode which also influences the response of the sea surface temperature and velocity.

Spin-up in this case resembles that described in Philander and Pacanowski (1980) but the presence of only two discrete baroclinic modes simplifies the resulting evolution. Initially, the Yoshida jet is excited in both modes, although it is stronger in the second baroclinic mode. The westward forcing drives the lower-layer current westward in mode 1 and eastward in mode 2. The acceleration associated with the firstmode Yoshida jet stops in the wake of the Kelvin wave front. The second-mode Yoshida jet continues, resulting in an apparent acceleration. Eventually, the arrival of the second-mode Kelvin wave front from the western side of the basin stops this acceleration as well, and the flow ceases its acceleration. In linear theory, the arrival of the second-mode Kelvin wave should very nearly stop all flow in the two layers, by allowing a pressure gradient in the first layer to balance the wind driving, and by allowing the internal mass field to achieve exact compensation so that the pressure gradient vanishes in the second layer. This does not happen because modifications in the modal structure prevent this balance.

It is possible in a multi-layered, stratified model to obtain a no-flow solution, where the stress balances the pressure gradient in the top layer, while internal density readjustments allow for zero pressure gradients beneath the stressed layer (see Cane, 1979, p. 238). If the model includes some other physics that interferes with its ability to adjust the subsurface pressure gradient to zero then an equatorial undercurrent will be generated. In the model of Cane (1979) there is no density difference between the two layers but only the top layer feels the wind stress. Even in the case where nonlinearities are suppressed, there will be an undercurrent, albeit a weak one (see Cane, 1979). In the stratified model of McCreary (1981) vertical diffusion of heat destroys the buoyancy profile needed to maintain a zero pressure gradient, and an undercurrent results.

The present model has features similar to both Cane (1979) and McCreary (1981) models. The entrainment of momentum allows for a non-linear momentum balance as in Cane, while the entrainment of buoyancy prevents full equilibrium of the density field and contributes to the generation of the undercurrent. To assess the importance of the nonlinear term we will consider the vertical advection of 
momentum in the zonal momentum balance. Along the equator, the momentum balance is

$$
u_{t}+u u_{x}+w_{e} u_{z}=-p_{x}+\frac{\tau^{x}}{h_{1}} .
$$

Fig. 3 shows the time averaged values of $w_{e} u_{z}, p_{x}$ and $-\tau^{x} / h$ from Case 2 . These values are arrived at by averaging over the last 80 days of the run. As can be seen, the vertical advection of momentum plays an important role in the balance. This model has no explicit vertical diffusion of heat or momentum; what diffusion there is results from mixed layer entrainment and this is closely related to vertical velocity. In the steady state, the entrainment rate and upwelling velocity are equal and the discussion following (4.2) suggests how $w_{e} u_{z}$ may be interpreted as a sum of diffusion and inviscid vertical advection. Either process alone would be sufficient to permit an undercurrent. McCreary rightly emphasizes the importance of vertical diffusion. In his model the vertical advection term is artificially suppressed although it is comparable to the retained terms. While either process alone would be sufficient to permit an undercurrent, a model which includes both in a realistic way leads to the conclusion that on the equator diffusion and vertical advection are inevitably related and both are non-negligible components of undercurrent dynamics.

Initially, the equatorial upwelling gives rise to a shallowing of the mixed layer. Since this shallowing is not accompanied by strong entrainment, the SST remains unchanged. After about 10 days the mixed

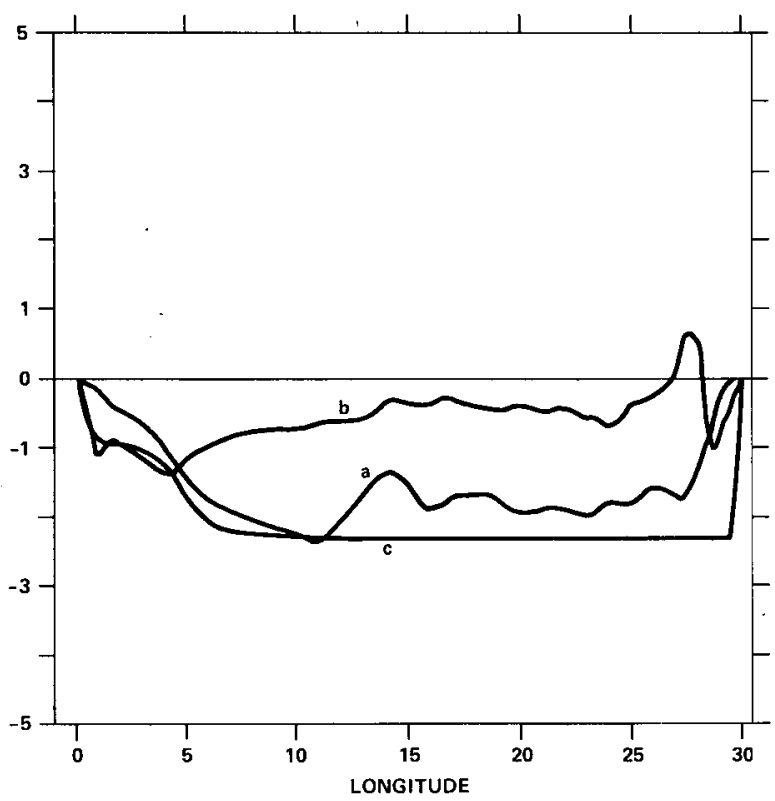

FIG. 3. Time-averaged values of the equatorial zonal momentum budget in the mixed layer. (a) $w_{e} \partial u / \partial z$, (b) $\partial p / \partial x$, and (c) $-\tau^{x} / h_{1}$. Averages taken from days 240 to 320 of Case 2 .
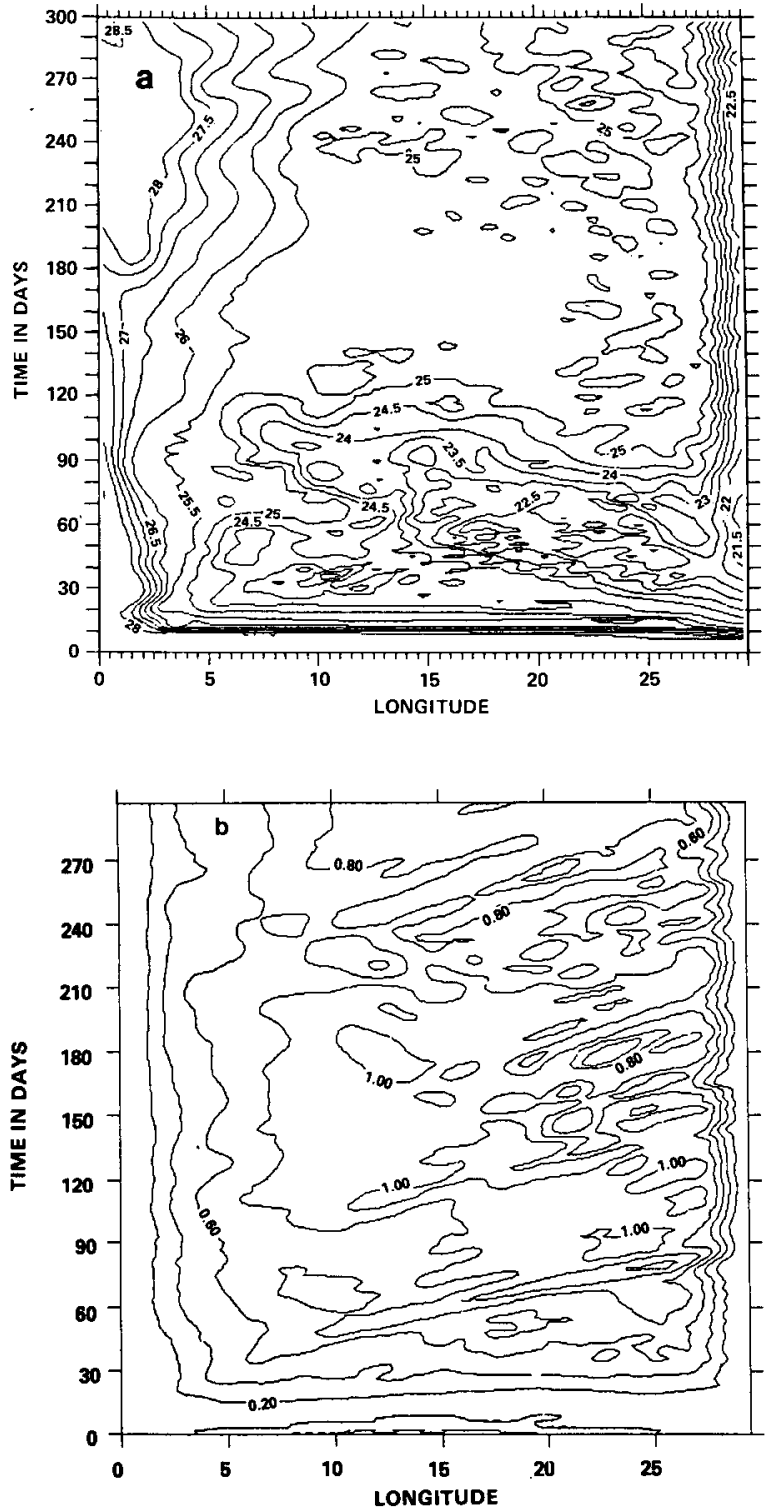

FIG. 4. Time-longitude section of (a) sea-surface temperature and (b) undercurrent speed along the equator from Case 2. Contour intervals are $0.5^{\circ} \mathrm{C}$ and $0.10 \mathrm{~m} \mathrm{~s}^{-1}$.

layer hits its minimum depth of $20 \mathrm{~m}$. Subsequent upwelling may be equated to entrainment, resulting in a rapid and strong cooling of SST all along the equator, which spreads out slowly. Fig. 4 is a timelongitude section of SST along the equator, where this rapid cooling in SST can be seen after 10 days. After this, stronger cooling is seen at the eastern end of the basin, due to the stronger upwelling and tilting of the isotherms in the second layer (Fig. 5).

\section{b. Frontogenesis}

By day 40 there is a band of shallow, cold water extending completely across the basin, within $3-4^{\circ}$ 


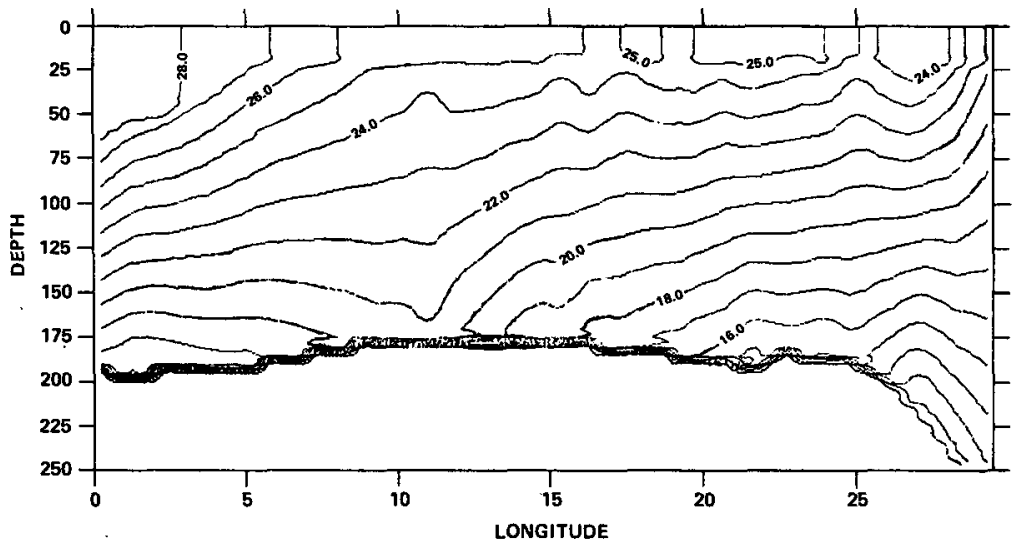

FiG. 5. Equatorial longitude-depth section of temperature at day 280 from Case 2. Contour interval $1^{\circ} \mathrm{C}$.

of the equator. In Fig. 6a, b, this shallow, cold region is seen to be bounded by a sharp front in both mixed layer depth and SST. This demonstrates one of the salient features of the interaction of ocean dynamics and mixed layer physics: when ocean dynamics produces upwelling, the equilibrium depth of the mixed layer changes from the diagnostic depth, $h_{\infty}$, to a value approximately one half of this depth.

First consider the situation where $w \geqslant 0$ : downwelling or no vertical motion. In this case, the mixed layer depth is determined by (3.5):

$$
h_{1}=\frac{2 m_{s} u_{*}^{3}}{\left(B_{0}+2 \epsilon_{0}\right)} \equiv h_{\infty}
$$

that is, $h_{1}$ is the diagnostic depth. Now assume that the mixed layer depth is in equilibrium in a upwelling
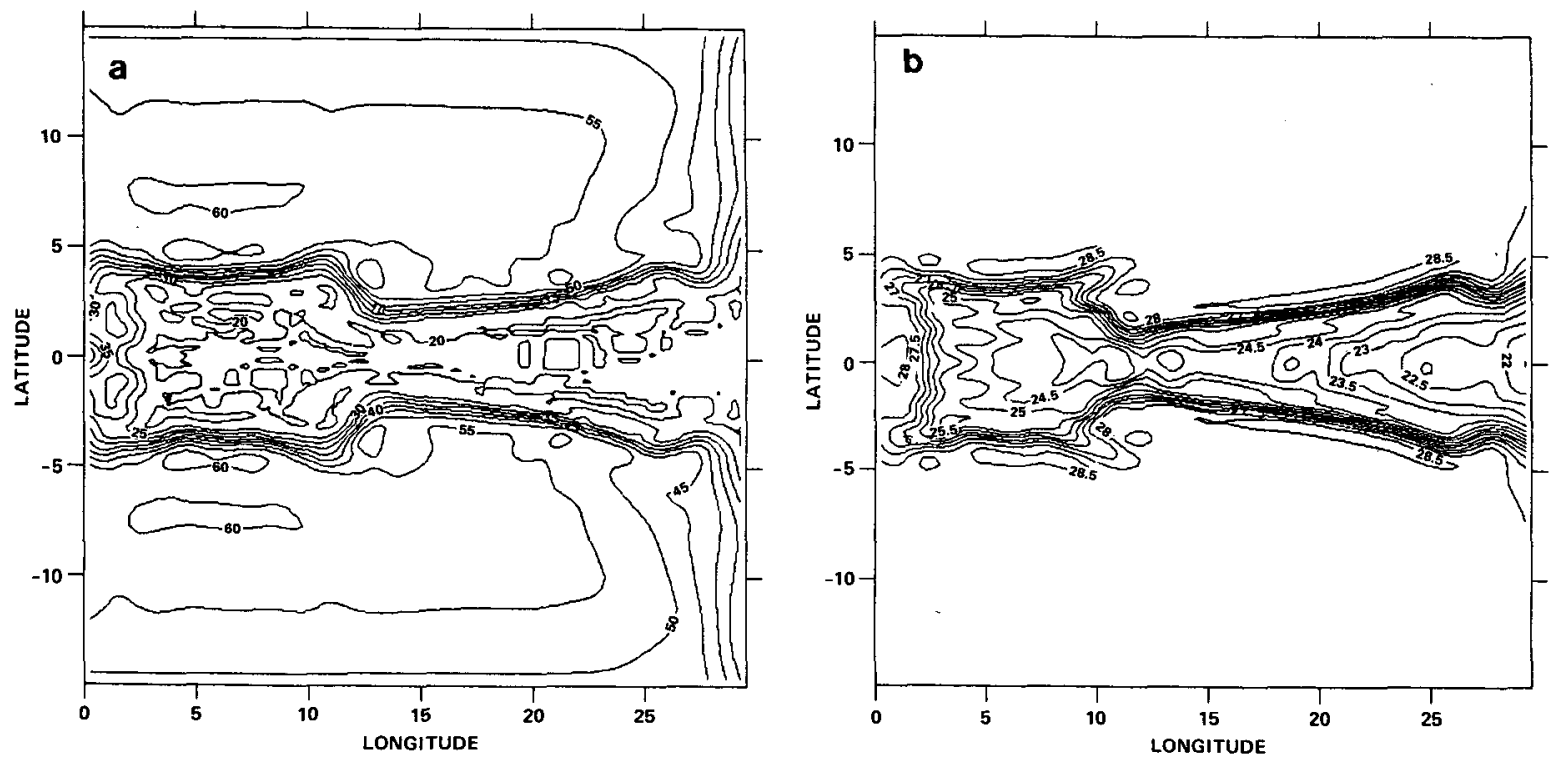

regime; hence $w_{e}=w$ [cf. Eq. (2.20)]. In order to maintain equilibrium the cooling caused by entrainment into the mixed layer must be balanced by surface heating: recasting the temperature equation (2.21) in terms of buoyancy,

$$
w_{e} \delta b=B_{0} .
$$

Neglecting the kinetic energy and penetrating radiation terms in (2.21), the mixed layer equation becomes

$$
w_{e} h_{1} \delta b=2 m_{s} u_{*}^{3}-\left(B_{0}+2 \epsilon_{0}\right) h_{1} .
$$

Using (4.6) on the left of (4.7), we obtain the equilibrium depth:

$$
h_{1 e}=\frac{m_{s} u_{*}^{3}}{\left(B_{0}+\epsilon_{0}\right)} \approx \frac{h_{\infty}}{2} .
$$

FIG. 6. (a) Mixed layer depth and (b) sea surface temperature after 40 days, from Case 2. Contour intervals of $5 \mathrm{~m}$ and $0.5^{\circ} \mathrm{C}$. 
At some distance from the equator there is a transition from the equatorial upwelling regime to an Ekman drift regime where the vertical velocity is downward. The preceding argument shows that in a quasiequilibrium situation there will be an abrupt change in mixed layer depth at the latitude where $w=0$, even though the change in the vertical velocity is relatively smooth.

An additional steepening effect arises because of our interactive heat flux law. As the upwelling cools the water, the heat flux increases [cf. (4.3)]. This decreases the diagnostic depth on the equatorward side of $w=0$. This effect occurs quite strongly in this model, changing $h_{\infty}$ from 54 to $18 \mathrm{~m}$ across the temperature front. In nature, the heat flux into the ocean also tends to increase with increased air-sea temperature difference, although other factors may modify the specifics.

The temperature front forms as a consequence of the jump in mixed layer depth at the latitude $y_{0}$ where $w=0$. Suppose that in the vicinity of $y_{0}$ the meridional velocity in the surface layer is just the Ekman velocity:

$$
v_{1}=\frac{-\tau^{x}}{\left(f h_{1}\right)}
$$

Obviously, the step in $h_{1}$ at $y_{0}$ results in an abrupt change in $v_{1}$; equatorward of $y_{0}$ there is strong advective cooling while poleward of $y_{0}$ the current is too slow to noticeably alter the SST. The sharp change in SST at $y_{0}$ results from the differential advection across the mixed layer depth front.

The preceding argument implies that if the mixed layer depth were held constant, the temperature front would not appear. Case 3 was run with the mixed layer depth fixed at $25 \mathrm{~m}$, but the initial thermal profile agreed with that used in Case 2. Upwelling and entrainment allowed subsequent evolution of the SST. In this case SST spreads much more rapidly and evenly. Fig. 7a shows the pattern at day 40 , where the regular spread in SST is visible (compare with Fig. $6 a)$. At the equator, the mixed layer treatments in the two cases are essentially similar ( $h=h_{\min }$ in Case 2; $h=h_{\text {fix }}$ in Case 3), and hence the SST and undercurrent behavior is quite similar.

The front in Case 2 continued to migrate poleward as more mass is upwelled at the equator, until it eventually becomes unstable and breaks down into a series of regular eddies centered at $4-5^{\circ}$ latitude. SST and mixed layer depth at 280 days are shown in Figs. 8a, $\mathrm{b}$, from which $800-\mathrm{km}$ waves can be clearly seen. A time-latitude plot of SST through the center of the basin (Fig. 9) shows the breakdown of the front at about 100 days followed by a very regular progression of waves through the remainder of the run. These waves appear similar to those found by Semtner and Holland (1980). While only suggestive, mid-latitude
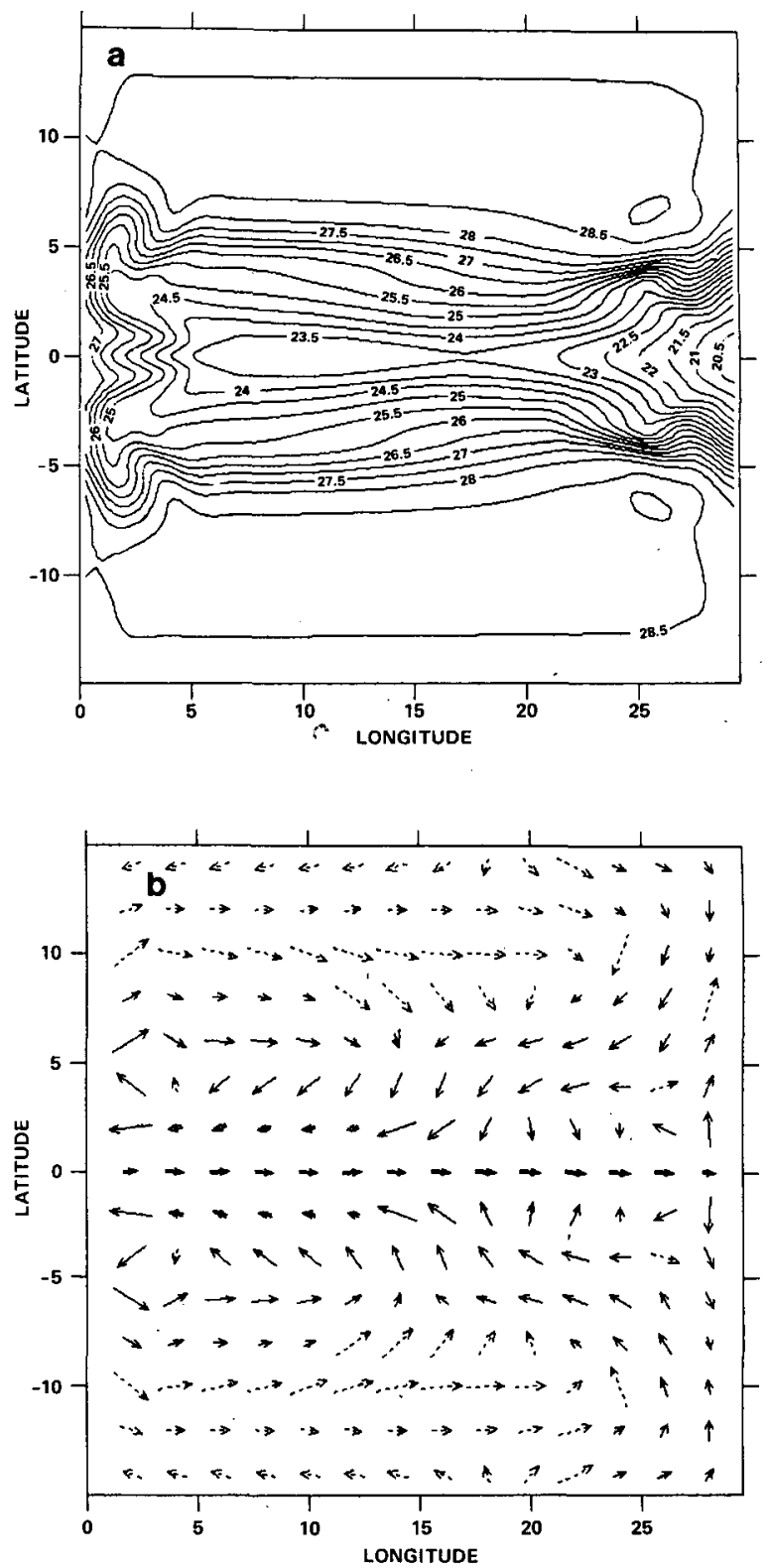

FiG. 7. (a) Sea surface temperature and (b) second layer velocity at day 40 of Case 3a. The mixed layer was fixed at $25 \mathrm{~m}$. Arrow length is proportional to speed-dashed arrows are 0.2 to $2 \mathrm{~cm} \mathrm{~s}^{-1}$, single arrows 2 to $20 \mathrm{~cm} \mathrm{~s}^{-1}$, double arrows 20 to $200 \mathrm{~cm} \mathrm{~s}^{-1}$.

analysis of the baroclinic instability of the two and one-half layer system on a $\beta$-plane centered at $5^{\circ}$ (Schopf, 1981) indicates a possibility for baroclinic instability with a maximum growth rate for waves of $780 \mathrm{~km}$.

An important feature of the response is the coupling of the thermal and velocity fields. The propagation of the second-mode Kelvin wave seen in Fig. $4 \mathrm{~b}$ is quite slow, similar to the slowing of the waves seen in Philander and Pacanowski (1980) in their non-linear case with $\tau^{x}=-0.5$. While the speed is 

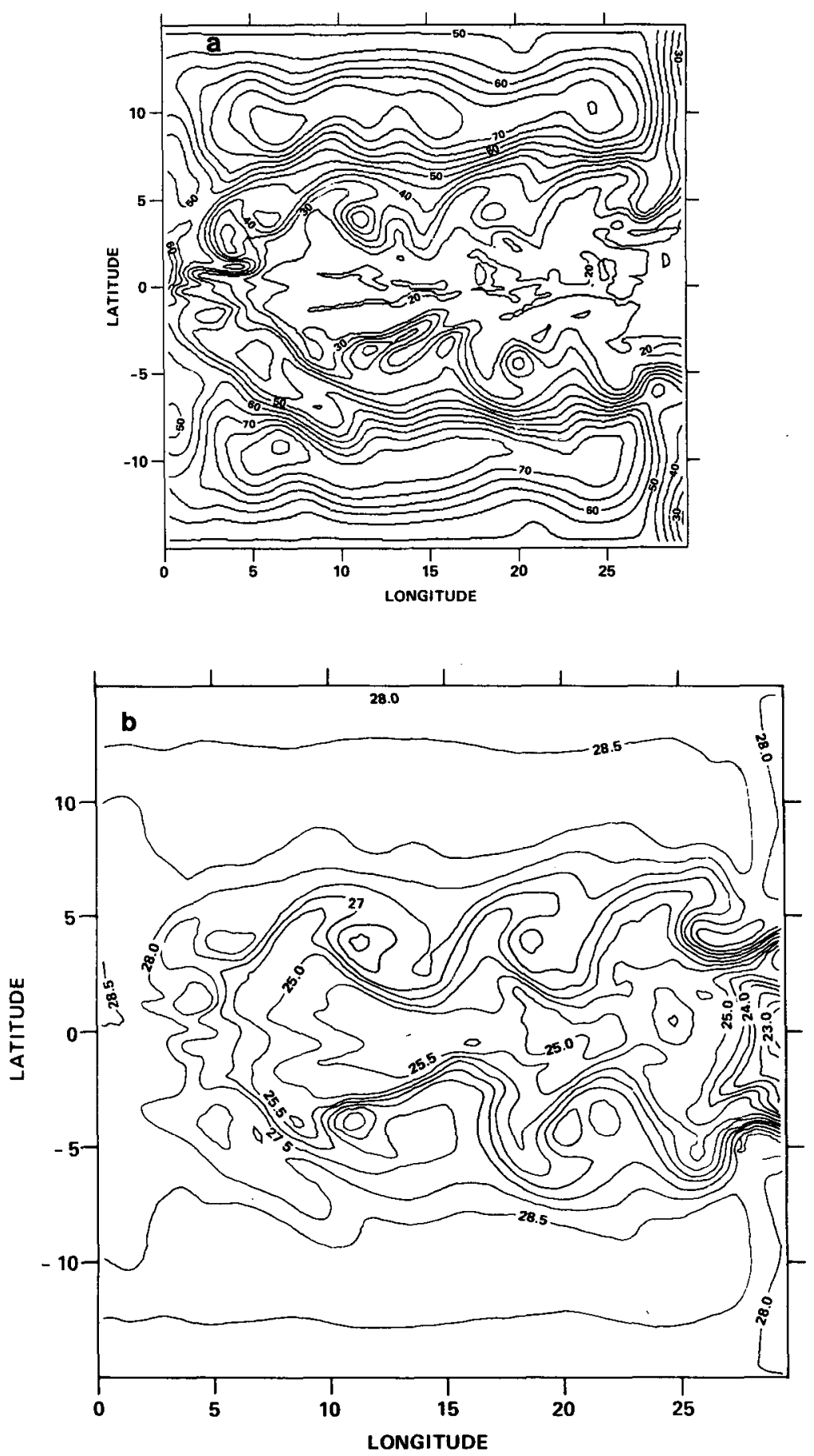

FIG. 8. (a) Mixed layer depth and (b) sea surface temperature after $\mathbf{2 8 0}$ days, from Case 2.

certainly affected by the presence of strong currents, it appears that in the present case this slowing is strongly related to a change in the stratification brought about by upwelling and mixing of the water in the vertical. The initial thermal profile led to a linear second-mode speed of $0.77 \mathrm{~m} \mathrm{~s}^{-1}$. Examining the equatorial thermal structure at $\mathbf{4 0}$ days into the run, we note a $4^{\circ} \mathrm{C}$ cooling of the surface water and an upward movement of isotherms in the second layer. This reduced buoyancy difference between the two layers results in a second-mode wave speed of about $0.42 \mathrm{~m} \mathrm{~s}^{-1}$. This analytic approximation for 


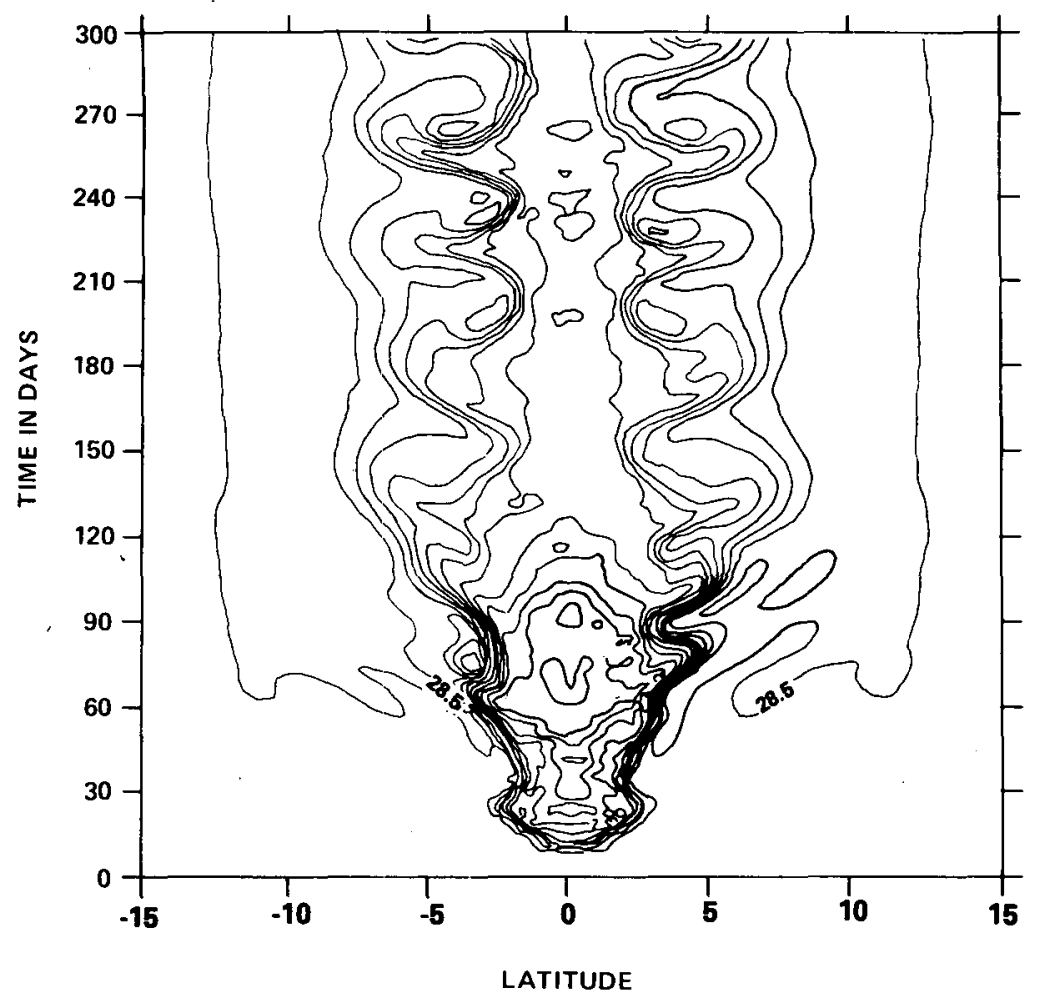

FIG. 9. Time-latitude section of sea surface temperature along the middle meridian of the basin from Case 2. Contour interval of $0.5^{\circ} \mathrm{C}$.

the second mode speed agrees very closely with the wave speed indicated in the time-space section in Fig. 4b.

\section{c. Response to southerly wind stress}

When the upper ocean is driven by uniform southerly winds, the linear response is anti-symmetric in layer thickness and zonal velocity and symmetric in meridional velocity. Thus, the upwelling induced along the eastern wall south of the equator is matched by downwelling to the north. Similarly there is an upwelling/downwelling pair along the western wall, with the upwelling to the north. The symmetry of the response is altered significantly by the nonlinearity of the entrainment/detrainment process, and by advective effects. With realistic mixed layer physics the sea surface temperature is not changed by detrainment, while it is cooled by entrainment. Thus the influence of downwelling (which in a steady state leads to detrainment) is not the complement of upwelling.

Case 4 was run as in Case 2, but with $\tau^{x}=0$ and $\tau^{y}=0.465$ dyn $\mathrm{cm}^{-2}$. The pattern of SST observed at 160 days in Fig. 10 shows that while the surface water has cooled south of the eastern end of the equator, it has not warmed to the north. In fact, no signature of the downwelling is visible in the SST.
While this result is not universal, as we shall show, it demonstrates clearly the differences between SST and PHA, and the difficulty which arises when attempting to interpret one in terms of the other.

Since the entrainment/detrainment process does conserve total heat and mass in the two layers, it affects the first baroclinic mode much less than the second. Those fields which are particularly sensitive to mode 1 will tend to maintain the linear symmetries. For example, the surface elevation or heat content of the two layers should remain asymmetric. The surface elevation is given by

$$
\zeta=\left(b_{1} h_{1}+b_{2} h_{2}\right) g^{-1},
$$

and is thus related to the upper ocean heat content due to the linear equation of state:

$$
\sum q=\left[\left(T_{1}-T_{r}\right) h_{1}+\left(T_{2}-T_{r}\right) h_{2}\right]=\zeta \alpha^{-1} \text {. }
$$

The second mode influences the vertical motion at the base of the mixed layer, and hence the entrainment/detrainment and changes in SST. Fig. 10 verifies the much stronger "anti-symmetry" in surface elevation than in SST.

\section{d. Response to southeasterly wind stress}

The lack of symmetry in SST due to the nonlinear nature of the entrainment/detrainment process does 

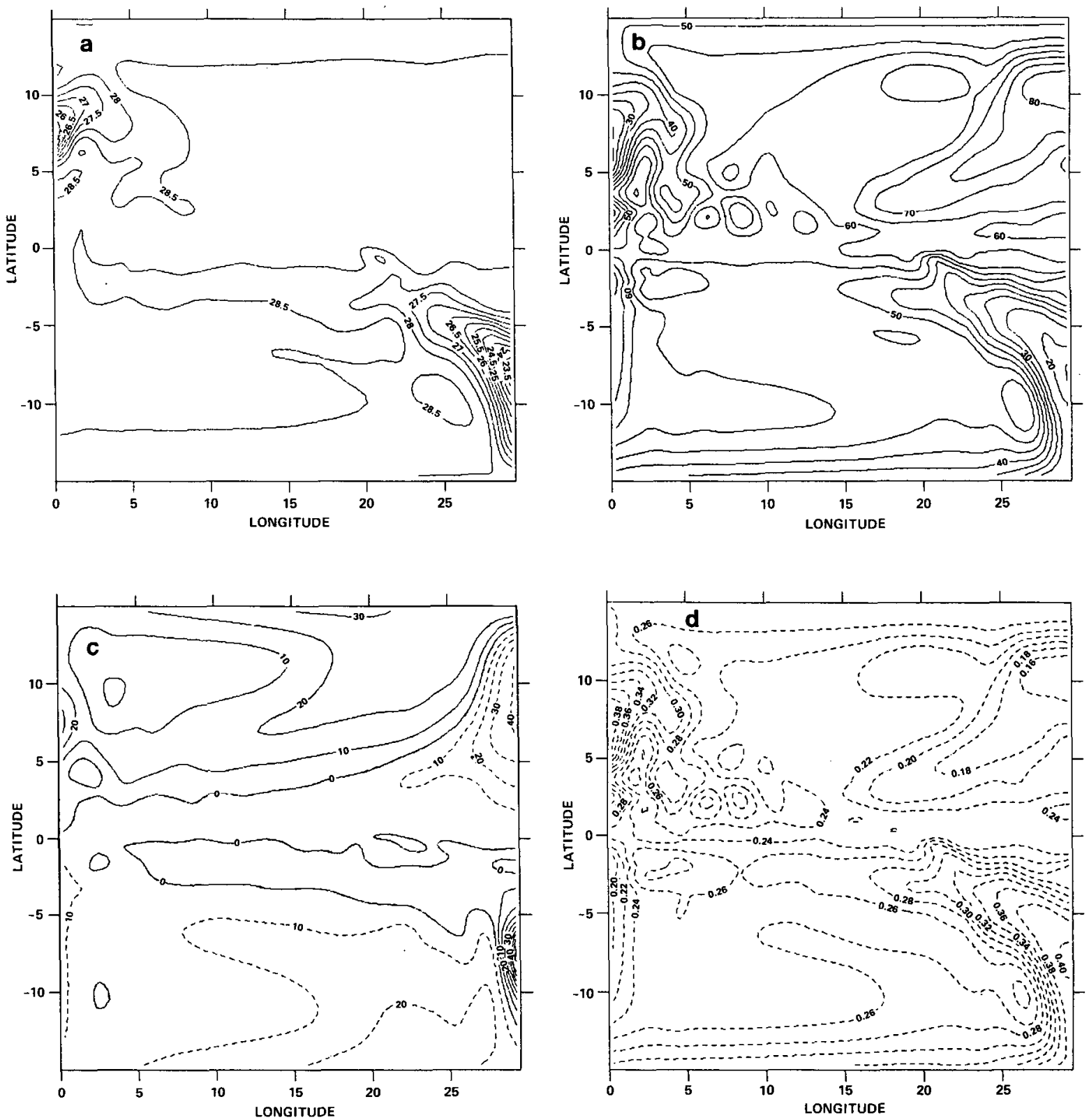

FIG. 10. (a) Sea surface temperature, (b) mixed layer depth, (c) pycnocline height anomaly and (d) surface elevation at day 160 of Case 4 , southerly wind driving.

not always hold. The most significant nonlinearity in the temperature equation occurs in the neighborhood of $w_{e}=0$, where the contribution due to vertical advective flux encounters a step. Away from $w_{e}=0$, the flux is in fact quite linear, as is reflected in the model results discussed below.

In the case with easterly wind there is upwelling all along the equator and slightly north and south along the eastern wall. Case 5 was run as Cases 2 and 4 , but with the forcing specified as the superposition of the two $\left(\tau^{x}=-0.465\right.$ dyn $\mathrm{cm}^{-2}, \tau^{y}=0.465$ dyn $\mathrm{cm}^{-2}$ ). The results appear as a near superposition of the two cases, but here the downwelling associated with the southerly component only reduces the upwelling due to the easterlies, thus decreasing the cooling due to entrainment (i.e. the perturbation effect is warming). Fig. 11 shows the SST and surface height in the southeasterly wind case and the difference between the southeasterly and easterly cases. In Fig. 11 c, a relative warming can be seen north of the equator at the eastern boundary.

Other features of southeasterly driving are also present in the model results. The equatorial upwelling is shifted to windward, as seen in a temperature sec- 

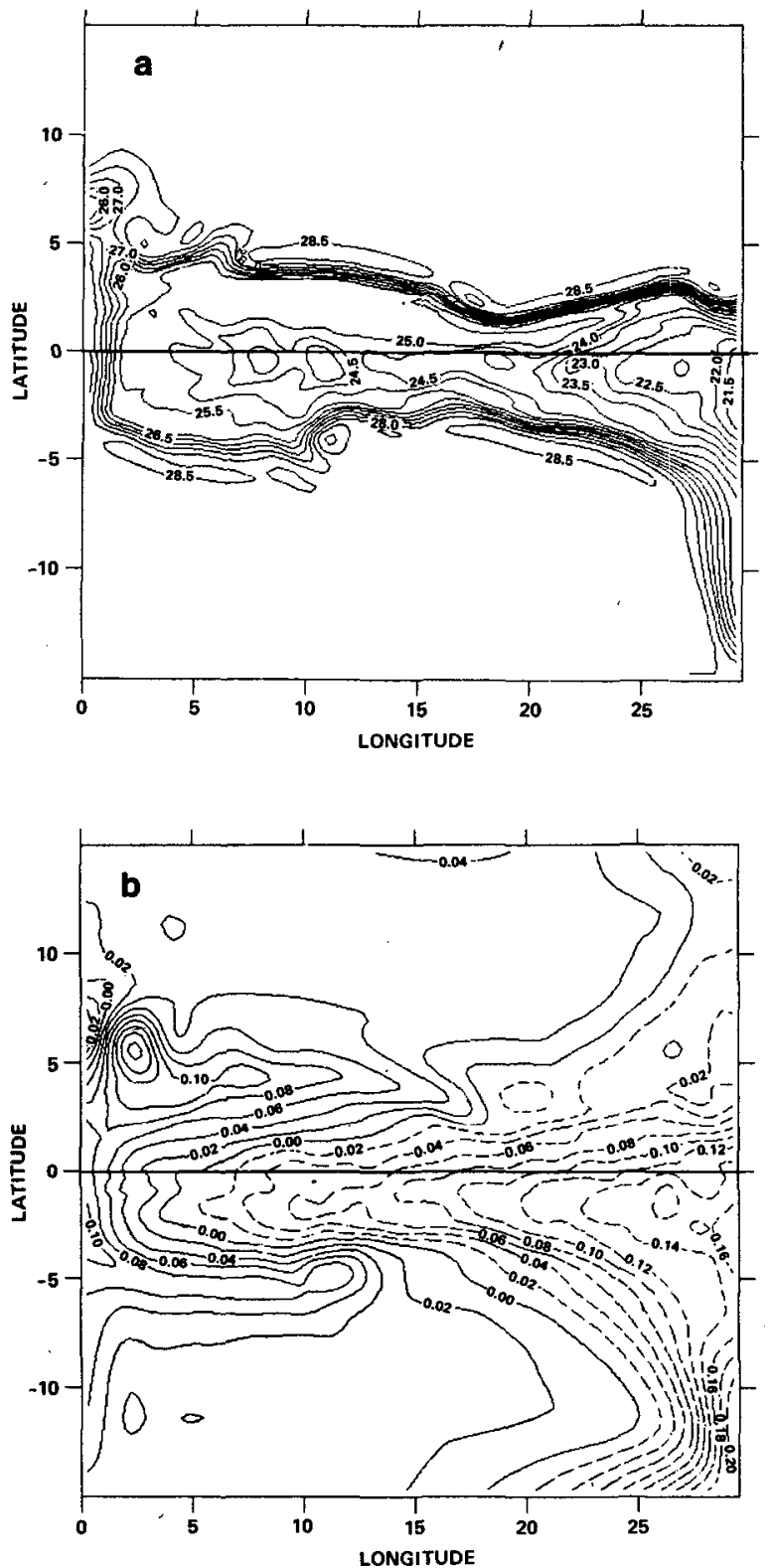

tion across the middle of the basin in Fig. 12. The maximum upwelling is now at $3^{\circ} \mathrm{S}$ while the undercurrent core is only moved about $1.5^{\circ}$. Of the cases presented here, this forcing and its SST response most resemble the observations in the eastern Pacific and Atlantic.

\section{Discussion}

There are two parts to the work described in this paper: 1) the development of a new numerical model including both primitive equation dynamics and mixed layer physics; 2) a study of the interplay of dynamics and thermodynamics in equatorial oceans.

The numerical model consists of two layers in the

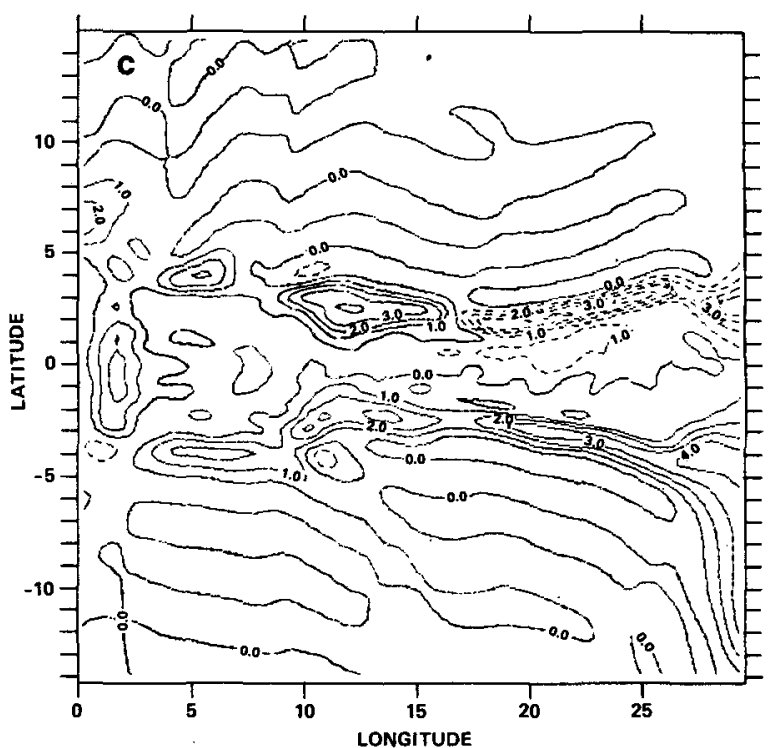

Fig. 11. (a) Sea surface temperature and (b) surface height at day 40 from Case 5 , southeasterly wind. Differences between Case 5 and Case 2 are shown in (c) for SST.

vertical, each of which is governed by primitive equation dynamics. The surface layer properties are also governed by mixed layer physics parameterized in terms of the turbulent kinetic energy balance in the manner of Kraus and Turner (1967). The mixed layer equations are solved with a quasi-analytic scheme that is accurate and efficient; it could be readily applied to other mixed layer studies. The numerical model employs a generalized vertical coordinate with the ocean mixed layer represented as a single layer for both dynamics and mixed layer physics. This treatment is efficient, accurate and internally consistent: no redundant quantities are calculated, the mixed layer depth is an explicit variable, and the same assumptions about the vertical structure of the mo- 


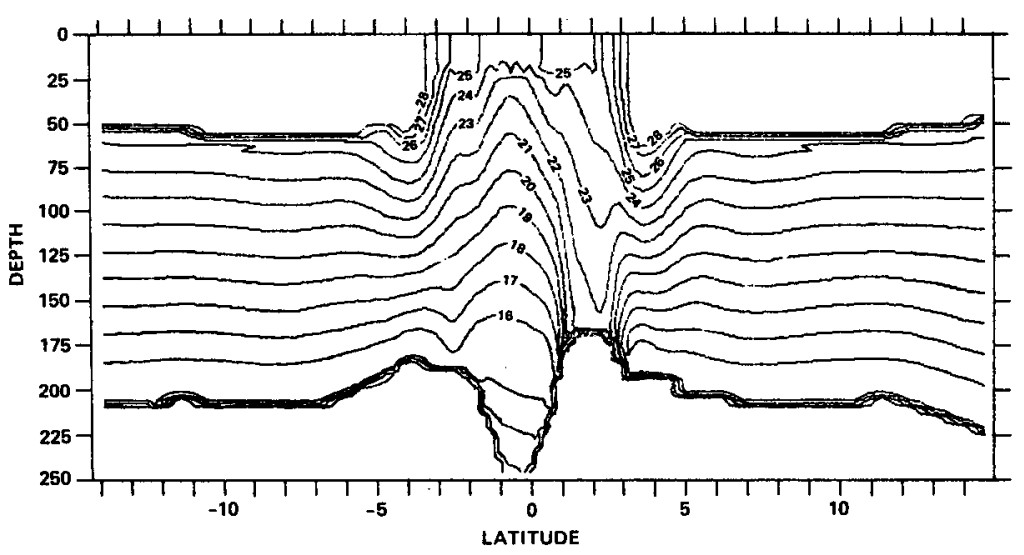

FIG. 12. Latitude-depth section of temperature along the middle of the basin at day 40 of Case 5 .

mentum and buoyancy fields apply to both the dynamics and mixed layer physics.

Models of mixed layer processes have achieved significant success in simulating the observed behavior of mixed layer variables, but our present understanding of mixed layer physics is inadequate to formulate an entirely satisfactory parameterization. For example, within regions of net surface cooling and convergent surface flow, the only process limiting deepening is dissipation, but dissipation within the mixed layer is not at all understood. Such shortcomings in mixed layer parameterizations necessarily limit our models' ability to simulate the upper ocean.

The greatest deficiencies of the model lie with the crude representation of the ocean just below the mixed layer. In the central Pacific there is substantial shear from the base of the mixed layer to the core of the undercurrent. The momentum entrained into the mixed layer would therefore be somewhat less than that simulated in the present model which permits no vertical shear within the second layer. In the western Pacific, the observed temperature is uniform to great depths but the currents are not, implying a shallower mixed layer than would be estimated from the temperature field. We have already pointed out that the limited thermal structure does not represent the detrainment process very well; under western Pacific conditions, our model immediately mixes detrained warm water downwards, artificially enhancing the temperature jump at the base of the mixed layer. Both of these problems could be readily alleviated by the inclusion of more vertical structure immediately below the mixed layer.

Increased vertical resolution could be added without altering the treatment of the mixed layer. The principal technical problem introduced by the model's mixed layer structure is the danger that inertia-gravity waves generated by rapid mixed layer excursions will destroy the calculation. The two layer version demonstrates that our numerical scheme handles it without resorting to excessive horizontal mixing, and a multilayer version should behave no differently. In any event, the configuration used here is sufficient for our present purposes.

In this, our initial set of experiments, we have focused on equatorial regions where dynamics are known to have a strong influence on SST. In fact, the vertical circulation at the equator is so vigorous that in the absence of surface heating the stratification is quickly $[O(100$ days)] eroded, and nothing inhibits the surface mixed layer from deepening down to the main thermocline. As a result the water column becomes homogeneous in both temperature and momentum, and the direct effect of the surface wind stress is felt at depth. This interfered with the usual equatorial dynamics and destroyed the equatorial undercurrent. Thus the primary role of surface heating was found to be maintenance of the stratification at the equator so that realistic dynamics would be possible. Its effects on SST and surface dynamic topography were less dramatic.

Once a reasonable buoyancy profile was maintained by surface heating, the model produced a vigorous equatorial undercurrent. Equatorial upwelling led to a non-linear transfer of energy from the second internal mode to the first, and this excludes the possibility of a no-motion solution for steady easterly winds. The resulting equatorial surface current system is maintained principally by a non-linear balance, with vertical advection of momentum balancing the wind driving.

It is common in equatorial studies to describe processes in terms of the baroclinic modes appropriate to linear dynamics. In our model there are only two vertical modes, the gravest being essentially uniform in velocity through the two active layers and the second changing sign at the base of the mixed layer. This second mode substitutes for a set of higher modes that would be present in the upper layers of real oceans. A principal use of the modal description is 
in explaining the ocean's response to a perturbation in the winds. The second mode has the larger vertical velocity at the base of the mixed layer and hence the larger effect on entrainment and SST. The first mode is the principal influence on sea surface elevation.

It is also of interest to consider the modifications of such linear modes by nonlinear effects. The nonlinear term that most influences the modal behavior is the vertical transfer of momentum between the mixed layer and the ocean below. It tends to reduce vertical gradients thus acting as a dissipation for the second mode by converting it into the first mode. There is little direct effect on the first mode because it has no vertical shear. Because the mixed layer is thin and the undercurrent is thick, the relative impact on the first mode by conversion from the second is small. Since the currents have more of a second mode structure, while surface dynamic topography is primarily associated with the first mode, the former is influenced by nonlinearity far more than the latter. It has been noted that in the nonlinear models of Cane (1979) and Philander and Pacanowski (1980), the adjustment of surface dynamic topography appears to be reasonably well described by linear dynamics but the evolution of the current system is not. The discussion presented here suggests why this is the case.

In our experiments the strongest upwelling and lowest temperatures occur at the eastern end of the equator, even when the winds are solely from the east. The eastern upwelling creates a zonal temperature gradient along the equator. In the Atlantic and $\mathrm{Pa}$ cific, the winds at the eastern end of the equator are primarily southerly and the lowest temperatures there result from the coastal upwelling south of the equator. Similar results were obtained in our experiments with southerly and southeasterly winds. The cold water upwelled at the eastern end is advected westward along the equator by the model's version of the South Equatorial Current. It is warmed by the surface heating as it travels so the zone of coldest temperatures does not extend across the basin.

Our calculations illustrate the point that the connection between changes in SST and in dynamic quantities such as sea surface elevation need not be straightforward. The strong asymmetry between upwelling and downwelling in their interaction with mixed-layer processes is a principal reason. Downwelling deepens the mixed layer but typically does not make the SST warmer. Upwelling tends to enhance entrainment by making the surface layer shallower and tends to decrease SST by bringing colder water closer to the surface where mixing into the surface layer is easier. The asymmetry between upwelling and downwelling shows clearly at the eastern end of the basin in the southerly wind case. The dynamic topography is approximately anti-symmetric about the equator, but the upwelling south of the equator sharply decreases the SST while the downwelling north of it leaves SST virtually unchanged.

In order to assess SST changes in response to wind field perturbations one must consider the changes in the upwelling/downwelling pattern. A modulation where $w>0$ is likely to lead to measurable SST changes, while a modulation of $w<0$ is not. In the vicinity of $w=0$, changes are particularly hard to predict because the perturbation can change the sign of $w$ inducing substantial changes in SST. In the initial response to easterly winds the equatorial upwelling rate exceeds the rate of deepening by turbulent mixing so the mixed layer shallows but with little entrainment, and hence almost no change in SST. Once the layer becomes shallow enough entrainment becomes strong and SST decreases rapidly. In the case of southeasterly winds the effects of the southerly component modulated the positive entrainment on both sides of the equator that arises from the easterly wind. The water south of the equator became colder and the water north of the equator became warmer (less cold), as compared against the results with easterlies alone.

In all cases where the mixed layer depth was allowed to change, the strong interaction of mixed layer physics and upwelling resulted in discontinuities in mixed layer properties. For example, with easterly winds equatorial upwelling creates a zone with a shallow mixed layer ending abruptly at the latitude where the vertical velocity goes to zero. Downwelling poleward of this latitude results in a much deeper surface layer with a reduced surface velocity. With a southerly wind coastal upwelling leads to qualitatively similar behavior. It was noted that in downwelling regions the mixed layer depth is approximately the diagnostic depth, while in upwelling regions it approaches one half the diagnostic depth. The depth disparity is enhanced by the tendency of the surface heat flux to be greater in the upwelling region where the SST is colder, thus decreasing the diagnostic depth there. Hence, even if the vertical velocity varies smoothly through the line where $w=0$, the mixed layer depth

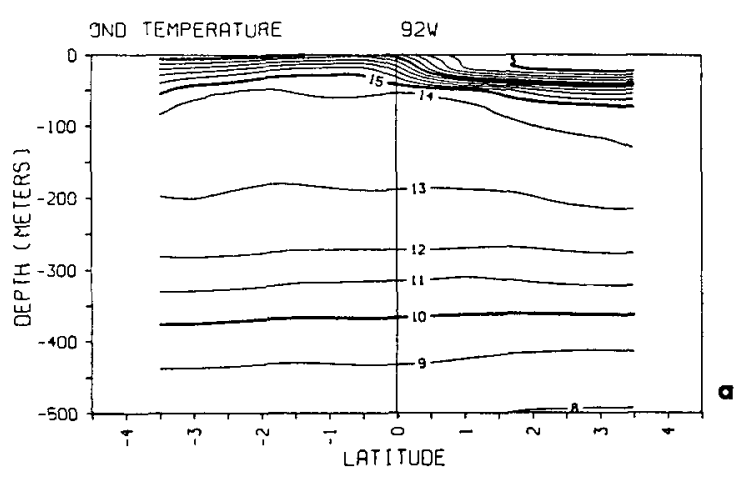

FIG. 13. Mean temperature section along $92^{\circ} \mathrm{W}$ for October-December from Lukas (1981). 
will be discontinuous there. Typically, the wind drives faster currents in the shallower surface layer, enhancing horizontal temperature advection on the shallow, cold side of the discontinuity. As a result, a surface temperature front is created. The Galapagos Front bears a strong resemblance to the model fronts (Fig. 13). South of it the water is cold, and the mixed layer is so shallow as to be almost non-existent; to the north of the narrow region of strong SST gradient, the surface mixed layer is 25 meters deep and is clearly defined.

Acknowledgments. Our thanks to Ed Harrison for valuable criticisms of an earlier version of this manuscript. We gratefully acknowledge the long-standing support and encouragement from Dr. Milton Halem of the Goddard Modeling and Simulation Facility. Wally Gross and Dennis Meyers provided valuable programming support. MAC's participation was supported by NASA Grant NGR 22-009727 and NSF Grant OCE-7922046 to the Massachusetts Institute of Technology.

\section{REFERENCES}

Adamec, D., R. L. Elsberry, R. W. Garwood, Jr. and R. L. Haney, 1981: An embedded mixed layer-ocean circulation model. Dyn. Atmos. Oceans, 6, 69-97.

Arakawa, A., 1972: Design of the UCLA general circulation model. Numerical Simulation of Weather and Climate, Dept. of Meteor., University of California Los Angeles, Tech. Rep. No. 7, $116 \mathrm{pp}$.

Busalacchi, A. J., and J. J. O'Brien, 1981: Interannual variability of the equatorial Pacific in the 1960's. J. Geophys. Res., 86, 10 901-10 907.

Cane, M. A., 1979: The response of an equatorial ocean to simple wind-stress patterns, Parts I and II. J. Mar. Res., 37, 233-299.

Davis, R. E., R. DeSzoeke, D. Halpern and P. Niiler, 1981: Variability in the upper ocean during MILE. Part I: Heat and momentum balances. Deep Sea Res., 28, 1427-1452.

Denman, K. L., and M. Miyake, 1973: Upper layer modification at Ocean Station Papa: Observations and simulation. J. Phys. Oceanogr., 3, 185-196.

Garwood, R. W., Jr., 1979: Air-sea interaction and dynamics of the surface mixed layer. Rev. Geophys. Space Phys., 17, 15071524.

Halpern, D., 1980: Variability of near-surface currents in the Atlantic North Equatorial countercurrent during GATE. $J$. Phys. Oceanogr., 10, 1213-1220.

Haney, R. L., 1971: Surface thermal boundary condition for ocean circulation models. J. Phys. Oceanogr., 1, 241-248.
Israeli, M., and D. Gottlieb, 1974: On the stability of the N-cycle scheme of Lorenz. Mon. Wea. Rev., 102, 254-256.

Kalnay-Rivas, E., and D. Hoitsma, 1980: The effect of accuracy, conservation and filtering on numerical weather forecasting. Proc. Fourth Conf. Numerical Weather Prediction, Silver Spring, Amer. Meteor. Soc., 410 pp.

Kasahara, A., 1974: Various vertical coordinate systems used for numerical weather prediction. Mon. Wea. Rev., 102, 509-522.

Kraus, E. B., and J. S. Turner, 1967: A one-dimensional model of the seasonal thermocline. II: The general theory and its consequences. Tellus, 19, 98-109.

Kim, J.-W., 1976: A generalized bulk model of the oceanic mixed layer. J. Phys. Oceanogr., 6, 686-695.

Lorenz, E., 1971: An N-cycle time-differencing scheme for stepwise numerical integration. Mon. Wea. Rev., 99, 644-648.

Lukas, R., 1981: The termination of the equatorial undercurrent in the eastern Pacific. Ph.D. thesis, University of Hawaii, 127 pp.

Marchuk, 1974: Numerical Methods in Weather Prediction, K. N. Tritogoff and V. R. Lamb, Eds. Academic Press, 277 pp.

McCreary, J. P., Jr., 1981: A linear stratified ocean model of the equatorial undercurrent. Phil. Trans. Roy. Soc. London, A298, 603-625.

Niiler, P. P., and E. B. Kraus, 1977: One dimensional models of the upper ocean. Modeling and Prediction of the Upper Layers of the Ocean, E. B. Kraus, Ed., Pergamon Press, 143-172.

Pacanowski, R., and S. G. H. Philander, 1981: Parameterization of vertical mixing in numerical models of tropical oceans. $J$. Phys. Oceanogr., 11, 1443-1451.

Philander, S. G. H., and R. Pacanowski, 1980: The generation of equatorial currents. J. Geophys. Res., 85, 1123-1136.

Pollard, R. T., P. B. Rhines and R. O. R. Y. Thompson, 1973: The deepening of the wind-mixed layer. Geophys. Fluid Dyn., 3, 381-404.

Pollard, D., 1982: The performance of an upper-ocean model coupled to an atmospheric GCM: Preliminary results. Rep. 31, Climate Res. Inst., Oregon State University, Corvallis, 33 pp.

Price, J. F., 1981: Upper ocean response to a hurricane. J. Phys. Oceanogr., 11, 153-175.

Schopf, P. S., 1981: Shallow baroclinic instability in the tropical oceans. Recent Progress in Equatorial Oceanography, J. P. McCreary, Jr., D. W. Moore and J. M. Witte, Eds., Nova University/N.Y.I.T. Press, 189-195.

Semtner, A. J., and W. R. Holland, 1980: Numerical simulation of equatorial ocean circulation. $J$. Phys. Oceanogr., 10, 667693.

Shapiro, R., 1970: Smoothing, filtering and boundary effects. Rev. Geophys. Space Phys., 8, 359-387.

Thompson, R. O. R. Y., 1976: Climatological numerical models of the surface mixed layer in the ocean. J. Phys. Oceanogr., 6, 496-503.

Woods, J. D., 1980: Diurnal and seasonal variation of convection in the wind-mixed layer of the ocean. Quart. J. Roy. Meteor. Soc., 106, 379-394.

Yoshida, 1959: A theory of the Cromwell Current and equatorial upwelling. J. Oceanogr. Soc. Japan, 15, 154-170. 